\title{
O problema de Monge-Kantorovich para duas medidas de probabilidade sobre um conjunto finito
}

\author{
Estéfano Alves de Souza
}

\author{
DisSERTAÇÃO APRESENTADA \\ $\mathrm{AO}$ \\ Instituto DE MatemÁtica e EstatísticA \\ DA \\ Universidade DE SÃo PAUlo \\ PARA \\ OBTENÇÃO DO TÍTULO \\ $\mathrm{DE}$ \\ Mestre EM CIÊNCIAS
}

Área de Concentração: Estatística

Orientador: Prof. Dr. Jefferson Antonio Galves

Durante o desenvolvimento deste trabalho o autor recebeu auxílio financeiro do CNPq

São Paulo, fevereiro de 2009 


\title{
O problema de Monge-Kantorovich para duas medidas de probabilidade sobre um conjunto finito
}

\author{
Este exemplar corresponde à redação \\ final da dissertação devidamente corrigida \\ e defendida por Estéfano Alves de Souza \\ e aprovada pela Comissão Julgadora.
}

Banca Examinadora:

- Prof. Dr. Jefferson Antonio Galves (orientador) - IME-USP.

- Profa. Dra. Florencia Graciela Leonardi - IME-USP.

- Profa. Dra. Nancy Lopes Garcia - UNICAMP. 


\section{Agradecimentos}

Primeiramente, gostaria de agradecer ao meu orientador, Antonio Galves, pela oportunidade que me deu e pelos ensinamentos e aconselhamentos que guardarei pelo resto da minha vida.

Agradeço também aos membros da banca pelas sugestões.

Ao CNPQ, pelo apoio financeiro nesses dois anos de Mestrado.

A todos que me ajudaram a chegar até este ponto de minha vida: amigos de longa data, amigos dos tempos do Bacharelado em Estatística, amigos que fiz nesses últimos dois anos, professores que me ensinaram coisas além do que apendemos normalmente nas escolas e universidades... Não vou

citar nomes porque, sem dúvida, cometerei injustiças ao esquecer alguns deles. Mas o fato é que essas pessoas têm meu eterno agradecimento - e saberão disso, se é que já não sabem.

A toda minha família: àqueles que ainda estão vivos e àqueles que já nos deixaram (alguns cedo até demais), mas que foram muito importantes na minha vida.

E finalmente, aos meus pais, Maria das Graças e Valdir, que sempre me apoiaram, independentemente das dificuldades que passamos neste longo caminho. 


\section{Resumo}

Apresentamos o problema do transporte ótimo de Monge-Kantorovich com duas medidas de probabilidade conhecidas e que possuem suporte em um conjunto de cardinalidade finita. O objetivo é determinar condições que permitam construir um acoplamento destas medidas que minimiza o valor esperado de uma função de custo conhecida e que assume valor nulo apenas nos elementos da diagonal.

Apresentamos também um resultado relacionado com a solução do problema de Monge-Kantorovich em espaços produto finitos quando conhecemos soluções para o problema nos espaços marginais.

Palavras-chave: Monge-Kantorovich, problema do transporte ótimo, acoplamento, Programação Linear. 


\section{Abstract}

We present the Monge-Kantorovich optimal problem with two known probability measures on a finite set. The objective is to obtain conditions that allow us to build a coupling of these measures that minimizes the expected value of a cost function that is known and is zero only on the diagonal elements.

We also present a result that is related with the solution of the Monge-Kantorovich problem in finite product spaces in the case that solutions to the problem in the marginal spaces are known.

Keywords: Monge-Kantorovich, optimal transportation problem, coupling, Linear Programming. 


\section{Sumário}

Lista de Notações $\quad$ ix

1 Introdução $\quad 1$

1.1 Monge e o problema do transporte ótimo . . . . . . . . . . . . . . . . . 1

1.2 A contribuição de Kantorovich . . . . . . . . . . . . . . . . . . 2

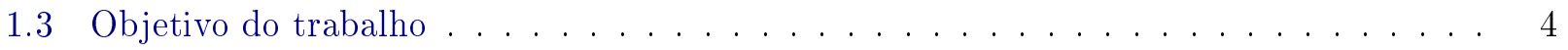

1.4 Contribuição do trabalho . . . . . . . . . . . . . . . . . 5

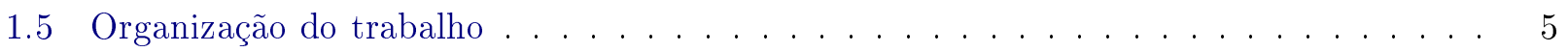

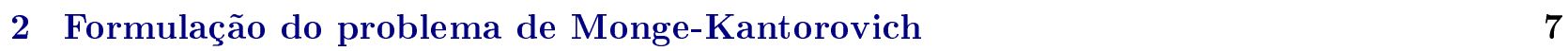

2.1 O problema de Monge-Kantorovich . . . . . . . . . . . . . . . . . . 7

2.2 A teoria de Kantorovich e Rubinstein . . . . . . . . . . . . . . . . . . . 10

2.3 O Teorema de Dobrushin $\ldots \ldots \ldots \ldots \ldots \ldots \ldots$

2.4 O Teorema da Dualidade . . . . . . . . . . . . . . . . . . . . 12

3 Construção de acoplamentos $\quad 15$

3.1 Acoplamentos e partições . . . . . . . . . . . . . . . . . . . 15

3.2 Uma condição necessária . . . . . . . . . . . . . . . . . . . . . . . 18

3.3 Acoplamentos em espaços produto . . . . . . . . . . . . . . . . . 22 
4 Exemplos $\quad 27$

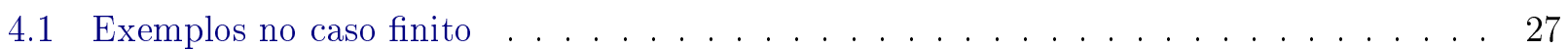

4.2 Exemplos no caso contínuo f . . . . . . . . . . . . . . . . . . 29

5 Programação Linear $\quad 33$

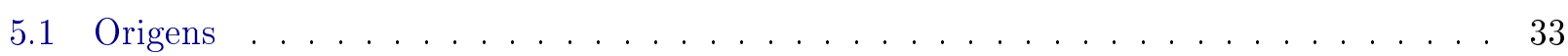

5.2 O problema do transporte . . . . . . . . . . . . . . . 35

5.3 Uma sugestão de algoritmo . . . . . . . . . . . . . . . . . . 37

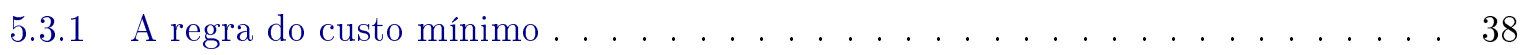

5.3 .2 Testando se uma solução é ótima . . . . . . . . . . . . . . . . . . 39

5.3.3 Melhorando uma solução básica . . . . . . . . . . . . . . . . . . . 40

5.3 .4 Exemplo de aplicação do algoritmo . . . . . . . . . . . . . . . 41

6 Considerações finais $\quad 47$

Referências Bibliográficas $\quad 49$ 


\section{Lista de Notações}

$\begin{array}{ll}\mathcal{B}\left(\mathbb{R}^{n}\right) & \sigma \text {-álgebra de Borel em } \mathbb{R}^{n} \\ \mathcal{L}^{1}(P) & \text { Conjunto das funções } f: \mathbb{R} \longrightarrow \mathbb{R} \text { tais que } \int_{-\infty}^{\infty}|f(x)| P(d x)<\infty \\ |A| & \text { Medida de Lebesgue (comprimento) de um intervalo } A \subseteq[0,1] \\ N_{p}(\mathbf{m}, \boldsymbol{\Sigma}) & \text { Distribuição Normal p-variada de vetor de médias } \mathbf{m} \text { e matriz de covariâncias } \boldsymbol{\Sigma} \\ |\boldsymbol{\Sigma}| & \text { Determinante de uma matriz quadrada } \boldsymbol{\Sigma} \\ \mathbf{x}^{T} & \text { Vetor transposto de } \mathbf{x} \in \mathbb{R}^{p} \\ \operatorname{tr}(\boldsymbol{\Sigma}) & \text { Traço de uma matriz quadrada } \boldsymbol{\Sigma}\end{array}$




\section{Capítulo 1}

\section{Introdução}

O problema do transporte ótimo de massa vem despertando o interesse de diversas áreas do conhecimento (como Geometria Diferencial, Programação Linear, Análise Funcional, Física e Teoria das Probabilidades) desde 1942, quando L.V. Kantorovich retomou o problema proposto inicialmente por G. Monge em 1781. Neste capítulo, apresentamos uma revisão histórica do problema, assim como o objetivo e a organização desta dissertação.

\subsection{Monge e o problema do transporte ótimo}

Em 1781, G. Monge propôs um problema de otimização [32] que virou uma espécie de protótipo para uma classe de problemas que surgiram posteriormente em diversos campos do conhecimento. Informalmente, tal problema consiste em transportar um volume fixo de terra de um ponto para outro, levando-se em conta que para cada unidade de terra a ser transportada existe uma posição final com determinado custo de transporte. Estamos interessados em obter uma forma de transportar a terra de modo que o custo total seja mínimo.

Descrevemos o problema em linguagem matemática da seguinte forma: Dados dois conjuntos $A, B \subset \mathbb{R}^{n}$ de volumes iguais, queremos encontrar uma função de transporte (ou simplesmente transporte) $t: A \longrightarrow B$ que preserva volume (ou seja, $t^{-1}(B)=A$ ) e que minimiza a seguinte função de otimização:

$$
C(t)=\int_{A} c(x, t(x)) d x
$$

onde $c(x, y)$ é o custo (não-negativo) de transportar $x \in A$ para $y \in B$.

Se $t^{*}$ é um transporte que minimiza $C(t)$, então é chamado de transporte ótimo ou, alterna- 
tivamente, solução ótima do problema. Monge considerou a função custo como sendo a distância Euclidiana em $\mathbb{R}^{n}$ (ou seja, $c(x, y)=|x-y|$ ), e demonstrou que a função de otimização $C(t)$ é não-linear na função de transporte $t$.

A Academia de Paris ofereceu, no século XIX, um prêmio pela solução do problema de Monge. Tal prêmio foi ganho por P. Appell, que estabeleceu algumas propriedades geométricas de transportes ótimos em $\mathbb{R}^{2}$ e em $\mathbb{R}^{3}$ (ver, por exemplo, [3]), ratificando algumas observações feitas por Monge em sua resenha original. Contudo, foi apenas em 1979 que surgiu o primeiro resultado geral de existência do transporte ótimo que soluciona o problema de Monge; este resultado é atribuído a Sudakov [40].

\subsection{A contribuição de Kantorovich}

Após os trabalhos de Monge e Appell sobre o problema do transporte ótimo, passou-se um longo tempo até que o assunto fosse retomado novamente. Entretanto, o russo L.V. Kantorovich escreveu em 1942 um artigo [22] no qual apresentou o problema de uma forma nova, resumida e mais acessível. Um detalhe importante é que Kantorovich não tinha conhecimento do trabalho de Monge quando escreveu seu artigo.

Na formulação de Kantorovich, o problema de obter uma função de transporte ótima é substituído pelo problema de encontrar um plano de transporte que minimiza o custo total. Kantorovich definiu a classe de planos de transporte como sendo o conjunto de todas as medidas de probabilidade sobre o respectivo espaço produto e com distribuiçôes marginais fixadas que representam, respectivamente, as distribuições de massa inicial e final. Maiores detalhes são apresentados no Capítulo 2.

Kantorovich, que estava ligado ao Governo da antiga União Soviética durante a Segunda Guerra Mundial, enuncia dois problemas práticos em seu artigo:

Problema 1: Uma rede de ferrovias conecta $m$ locais de produção $A_{1}, A_{2}, \ldots, A_{m}$ que produzem, respectivamente, $a_{1}, a_{2}, \ldots, a_{m}$ carregamentos de determinado bem de consumo a $n$ locais de consumo $B_{1}, B_{2}, \ldots, B_{n}$ que possuem demanda de $b_{1}, b_{2}, \ldots, b_{n}$ carregamentos, com a condição de que

$$
\sum_{i=1}^{m} a_{i}=\sum_{k=1}^{n} b_{k} .
$$

(Observe que essa condição é equivalente a atribuir distribuições de probabilidade para 
a produção e a demanda dos carregamentos.) Dado o custo $r_{i, k}$ envolvido em mover um carregamento de $A_{i}$ para $B_{k}$, encontrar um plano de transporte de carregamentos entre os locais de produção e os de consumo que minimiza o custo total de transporte.

Problema 2: Dadas as equações de uma superfície de terra $z=f(x, y)$ e $z=f_{1}(x, y)$ antes e depois de um nivelamento (ou seja, $\iint f(x, y) d x d y=\iint f_{1}(x, y) d x d y$ ) e o custo de transportar $1 m^{3}$ de terra de $(x, y)$ para $\left(x_{1}, y_{1}\right)$, obter um plano de transporte de massas de terra que apresenta o menor custo total de transporte.

De um lado, o primeiro problema está intimamente ligado à teoria matemática de Programação Linear (cujos passos iniciais durante o Século XX são atribuídos ao próprio Kantorovich no final da década de 1930) e a logística de guerra, uma vez que relaciona produção e demanda de determinado bem de consumo (por exemplo, armamento de guerra ou suprimentos) através de um plano de transporte com o menor custo de transporte possível, situação ideal em períodos de guerra. Por outro lado, o segundo problema lembra o problema original proposto por Monge, no sentido em que as equações de superfície determinam as distribuições inicial e final da massa de terra transportada.

Kantorovich escreveu em 1948 um artigo [23] no qual fez a conexão entre seu problema e o de Monge ao observar que sua formulação era, de fato, uma generalização da formulação proposta originalmente por Monge. A dificuldade existente no problema não-linear de Monge foi naturalmente substituída por um problema de otimização linear sobre um conjunto abstrato convexo. O resultado desta constatação foi colocar o problema na linha de frente da teoria de otimização linear e encorajar o desenvolvimento da Teoria da Dualidade para a solução do problema proposto por Kantorovich como uma ferramenta básica.

A partir do trabalho de Kantorovich, problemas relacionados e suas generalizações ficaram conhecidos como Problemas de Transporte de Massa de Monge-Kantorovich (ou, simplesmente, problemas de Monge-Kantorovich). Pela relação existente entre o problema de Monge-Kantorovich e a construção de variáveis aleatórias com marginais fixadas, diremos que um plano de transporte é um acoplamento de medidas de probabilidade.

Por suas contribuições posteriores para a teoria de transporte ótimo de recursos e Programação Linear, Kantorovich recebeu, juntamente com T.C. Koopmans, o Prêmio Nobel de Ciências Econômicas em 1975.

A formulação de Kantorovich para o problema do transporte ótimo e a teoria que foi gerada a 
partir da mesma tornaram o problema acessível às mais diversas áreas das Ciências Matemáticas e outros campos da Ciência. Enumeramos a seguir algumas referências nas Ciências Matemáticas:

- Análise Funcional: Kantorovich e Akilov [25]

- Teoria Probabilística: Dudley [10,11], Fréchet [14], Rachev [36] e Rüschendorf [38]

- Estatística: Gini [15], Hoeffding [18], Kemperman [30]

- Programação Linear: Anderson e Nash [2], Barnes e Hoffman [4], Hoffman [19]

- Teoria da Informação: Wasserstein [42]

Muitos problemas práticos relacionados a vários campos da Ciência levaram matemáticos a resolver problemas de Monge-Kantorovich, por exemplo, em:

- Física Estatística: Dobrushin [7]

- Controle de Qualidade: Jirina e Nedoma [20]

- Transporte: Dantzig e Ferguson [6]

- Econometria: Gretsky, Ostroy e Zame [16]

- Teoria das Filas: Rachev [35]

Rachev e Rüschendorf [37] é uma boa fonte tanto para um grande número de resultados como para outras referências relacionadas. Mais recentemente, trabalhos como o de Ambrosio et al. [1] retomam o problema de Monge-Kantorovich e sua importância na resolução de problemas em outras áreas, tais como Equações Diferenciais Parciais não-lineares e Geometria Riemaniana, além de apresentarem algumas desigualdades e resultados relevantes.

\subsection{Objetivo do trabalho}

Nosso objetivo principal é estudar o problema de Monge-Kantorovich no caso finito em duas situações diferentes: 
- Dadas duas medidas de probabilidade definidas em um mesmo conjunto de cardinalidade finita $S$, nosso objetivo é estabelecer condições que nos permitam construir explicitamente um plano de transporte que solucione o problema, no caso em que o custo assume valor nulo apenas nos elementos da diagonal de $S^{2}$.

- Apresentar um resultado relacionado com a solução do problema de Monge-Kantorovich em espaços produto finitos quando conhecemos soluções para o problema nos respectivos espaços marginais.

\subsection{Contribuição do trabalho}

Nossas duas contribuições principais são:

- Uma condição que um acoplamento deve satisfazer para que solucione o problema de MongeKantorovich no caso finito;

- Um resultado relacionado com a solução do problema de Monge-Kantorovich em espaços produto finitos.

\subsection{Organização do trabalho}

No Capítulo 2, damos a definição formal do problema de Monge-Kantorovich e revisamos alguns resultados básicos existentes na literatura sobre o assunto. O Capítulo 3 é dedicado à formulação de resultados que nos permitam obter acoplamentos que solucionam o problema no caso finito. No Capítulo 4, damos alguns exemplos nos quais o problema de Monge-Kantorovich pode ser solucionado de forma explícita. No Capítulo 5 discutimos a relação do problema com a Programação Linear e apresentamos um algoritmo que obtém uma solução ótima para o problema e, finalmente, o Capítulo 6 é dedicado às considerações finais e a sugestões para pesquisas futuras. 


\section{Capítulo 2}

\section{Formulação do problema de Monge-Kantorovich}

Neste capítulo, apresentamos a definição formal do problema de Monge-Kantorovich, passando pelos problemas propostos por Monge e Kantorovich separadamente, e alguns dos resultados mais comuns relacionados com o assunto.

\subsection{O problema de Monge-Kantorovich}

Vimos, na Seção 1.1, que o problema do transporte ótimo de Monge consiste em transportar determinada quantidade (distribuição) de massa de uma região a outra de tal forma que o custo do trabalho realizado seja mínimo.

Alguns autores (por exemplo, Ambrosio et al. [1, p. 123-124]) dão a seguinte formulação para o problema de Monge: Sejam $f, g: \mathbb{R}^{n} \longrightarrow[0, \infty[$ as funções que descrevem as distribuições inicial e final de massa, respectivamente, e satisfaçam a condição elementar de que a massa total a ser deslocada durante o processo não se altera:

$$
\int_{\mathbb{R}^{n}} f(x) d x=\int_{\mathbb{R}^{n}} g(y) d y
$$

Assumindo, sem perda de generalidade, que a massa total é igual a 1, dizemos que a transformação Borel-mensurável $t: \mathbb{R}^{n} \longrightarrow \mathbb{R}^{n}$ é um transporte se

$$
\int_{t^{-1}(E)} f(x) d x=\int_{E} g(y) d y, \quad \text { para todo Boreliano } E \in \mathcal{B}\left(\mathbb{R}^{n}\right)
$$


O problema consiste em minimizar o custo total $C(t)$ dentro da classe de transportes, ou seja, obter

$$
\inf \left\{C(t)=\int_{\mathbb{R}^{n}}|x-t(x)| f(x) d x: t \text { é um transporte }\right\} \text {. }
$$

Apresentando nessas condições, o problema de Monge pode ser estendido para outras direções extremamente úteis:

- Espaços mensuráveis gerais $(A, \mathcal{A})$ e $(B, \mathcal{B})$, com transformações mensuráveis $t: A \longrightarrow B$;

- Medidas de probabilidade gerais $\mu$ sobre $A$ e $\nu$ sobre $B$, para as quais a condição de compatibilidade de massa (2.2) se resume a

$$
\nu(E)=\mu\left(t^{-1}(E)\right) \quad \text { para todo conjunto mensurável } E \in \mathcal{B} ;
$$

- Funções de custo gerais: funções mensuráveis $c: A \times B \longrightarrow[0, \infty]$. Neste caso, o custo total a ser minimizado é

$$
W(t)=\int_{A} c(x, t(x)) \mu(x) d x
$$

Conforme salienta Ambrosio et al. [1, p. 124], mesmo em espaços Euclidianos, o problema da existência de transportes ótimos é longe de ser trivial, fato evidenciado pela não-linearidade, com respeito a $t$, da condição (2.4). Contudo, essa dificuldade começou a ser superada a partir de 1942, quando Kantorovich formulou sua versão do problema de transporte ótimo [22], na qual o interesse principal reside nos planos de transporte ao invés dos transportes, ou seja, o interesse passou a ser concentrado em medidas de probabilidade definidas em um espaço produto e com distribuições marginais fixadas.

Mais formalmente, o problema de Kantorovich pode ser enunciado da seguinte forma: Sejam $(S, \mathcal{B})$ um espaço mensurável e $\mathcal{P}(S)$ a classe composta por todas as medidas de probabilidade com suporte em $S$ e $\mathcal{B}$-mensuráveis. Dadas $\mu, \nu \in \mathcal{P}(S)$, definimos $\mathcal{M}(\mu, \nu)$ como o conjunto dos planos de transporte $Q$ definidos na $\sigma$-álgebra $\mathcal{B} \times \mathcal{B}$ e que têm $\mu$ e $\nu$ como marginais, ou seja,

$$
Q\left(A^{\prime}, S\right)=\mu\left(A^{\prime}\right) \text { e } Q\left(S, A^{\prime}\right)=\nu\left(A^{\prime}\right) \quad \text { para todo } A^{\prime} \in \mathcal{B}
$$


O problema de Kantorovich consiste em minimizar o custo total de transporte

$$
K(\mu, \nu):=\inf _{Q \in \mathcal{M}(\mu, \nu)} \iint_{S \times S} c(x, y) Q(d x, d y)
$$

ou, analogamente, obter um plano de transporte $Q^{*} \in \mathcal{M}(\mu, \nu)$ tal que o custo do processo realizado por este plano seja igual a $K(\mu, \nu)$. Nessas condições, dizemos que $Q^{*}$ é uma solução ótima (ou, simplesmente, solução) do problema de Kantorovich.

Observe que $\mathcal{M}(\mu, \nu)$ não é vazio (ver, por exemplo, Strassen [39]). Kantorovich definiu inicialmente o custo $c(x, y)$ de se mover de $x$ para $y$ como uma função real não-negativa arbitrária. Contudo, ao final de seu artigo, destacou que "é interessante estudar o problema quando $c(x, y)$ é uma distância sobre $S^{\prime \prime}$. De fato, a hipótese de que $(S, c)$ é um espaço métrico está associada a grande parte dos resultados que se sucederam ao artigo de Kantorovich. Rachev [34] é uma referência interessante para alguns desses resultados.

A formulação de Kantorovich, do ponto de vista probabilístico, é mais intuitiva que a formulação de Monge, uma vez que o problema passou a ser interpretado como uma minimização do valor esperado de uma função conhecida de duas variáveis aleatórias com distribuição conjunta determinada por marginais fixadas. Evidentemente, o problema de Kantorovich pode ser estendido para o caso em que $\mu \in \mathcal{P}(S)$ e $\nu \in \mathcal{P}\left(S^{\prime}\right)$, onde $S$ e $S^{\prime}$ são espaços diferentes.

Kantorovich escreveu em 1948 um artigo curto [23] na qual fez a conexão com o problema original proposto por Monge e afirmou que, sob certas condições associadas a (2.4), a solução ótima de seu problema também é solução do problema de Monge de modo que a formulação de Kantorovich generaliza a formulação de Monge. Em outras palavras: dada uma variável aleatória $X$ com lei $\mu$, o problema de Kantorovich é equivalente ao problema de obter uma função de transporte $t^{*}$ que satisfaça (2.4) e seja tal que a variável aleatória conjunta $(X, Y)$, onde $Y=t^{*}(X)$ tem lei $\nu$, minimize o custo esperado. Daí o fato do problema proposto por Kantorovich ser mais conhecido, desde então, como problema de Monge-Kantorovich.

Antes do final desta seção, apresentamos a seguinte

Definição 2.1.1 Definimos a quantidade $K(\mu, \nu)$ como sendo o minimo de Monge-Kantorovich. Além disso, diremos deste ponto em diante que $Q \in \mathcal{M}(\mu, \nu)$ é um acoplamento das medidas de probabilidade $\mu$ e $\nu$. 


\subsection{A teoria de Kantorovich e Rubinstein}

Kantorovich continuou seus estudos após sua formulação do problema do transporte ótimo e, juntamente com G.S. Rubinstein, introduziu o seguinte problema [28]: Dados $(S, \mathcal{B})$ um espaço mensurável e $\mu, \nu \in \mathcal{P}(S)$, obter

$$
\tilde{K}(\mu, \nu):=\inf _{Q^{\prime} \in \mathcal{M}^{*}(\mu, \nu)} \iint_{S \times S} c(x, y) Q^{\prime}(d x, d y),
$$

onde $\mathcal{M}^{*}(\mu, \nu)$ é o conjunto de todas as medidas finitas $Q^{\prime}$ sobre $\mathcal{B} \times \mathcal{B}$ tais que

$$
Q^{\prime}(A, S)-Q^{\prime}(S, A)=\mu(A)-\nu(A), \quad \text { para todo } A \in \mathcal{B}
$$

Em outras palavras, o problema consiste em minimizar o custo total de transporte por meio de planos de transporte de massa com diferenças de marginais fixadas. A esse problema, deu-se o nome de problema de Kantorovich-Rubinstein ou, como alguns autores sugerem, problema de transferência de massa (ver, por exemplo, Kemperman [29]).

Observe que $\mathcal{M}(\mu, \nu) \subseteq \mathcal{M}^{*}(\mu, \nu)$ e, portanto, $\mathcal{M}^{*}(\mu, \nu)$ não é vazio. Nessas condições, é natural procurarmos condições que nos permitam dizer quando os dois problemas são equivalentes. De fato, a conexão definitiva entre os dois problemas é feita pelo seguinte

Teorema 2.2.1 Os problemas de Monge-Kantorovich e Kantorovich-Rubinstein são equivalentes, ou seja,

$$
K(\mu, \nu)=\tilde{K}(\mu, \nu)
$$

apenas se c(x,y) é uma distância sobre $S$.

Este teorema (ver, por exemplo, Dudley e Neveu [12]) é um dos principais resultados que estão relacionados com a existência da solução ótima para o problema de Monge-Kantorovich (e, conseqüentemente, da solução ótima para o problema de Kantorovich-Rubinstein).

Sejam $(S, c)$ um espaço métrico e $\mu, \nu \in \mathcal{P}(S)$. Um dos resultados de Análise Funcional relacionado com o problema de Monge-Kantorovich (Dobrushin [8, p. 59-60]) é o seguinte

Teorema 2.2.2 A quantidade $K(\mu, \nu)$ define uma distância (denominada distância de Kantorovich) no espaço $\mathcal{P}_{0}(S) \subseteq \mathcal{P}(S)$ de medidas de probabilidade $\lambda$ tais que para algum (ou, equivalente- 
mente, qualquer) $x_{o} \in S$ vale que

$$
\int_{S} c\left(x, x_{o}\right) \lambda(d x)<\infty
$$

Outra distância em $\mathcal{P}_{0}(S)$ bem conhecida é a distância de Lipschitz:

$$
L(\mu, \nu)=\sup \left\{\int_{S} f(x)(\mu(d x)-\nu(d x)):|f(x)-f(y)| \leq c(x, y)\right\} .
$$

Finalmente, enunciamos outro resultado importante atribuído a Kantorovich e Rubinstein no final da década de 1950, que é o chamado Teorema de Kantorovich-Rubinstein [27]:

Teorema 2.2.3 Para quaisquer $\mu, \nu \in \mathcal{P}_{0}(S)$, vale que

$$
K(\mu, \nu)=L(\mu, \nu)
$$

Este teorema é base fundamental para o início, em termos de Teoria das Probabilidades, da chamada Teoria da Dualidade, que abriu definitivamente novas possibilidades para a solução de diversos problemas.

\subsection{O Teorema de Dobrushin}

Outro resultado importante relacionando distâncias entre medidas de probabilidades e o problema de Monge-Kantorovich é atribuído ao matemático russo R.L. Dobrushin. Antes de apresentarmos o resultado, damos a seguinte

Definição 2.3.1 Dadas $\mu$ e $\nu$ duas medidas de probabilidade sobre um espaço mensurável $(S, \mathcal{B})$, definimos a distância de variação total entre $\mu$ e $\nu$ como sendo

$$
\|\mu-\nu\|_{V T}=\sup _{B \in \mathcal{B}}|\mu(B)-\nu(B)| .
$$

Além disso, definimos a função custo $c: S^{2} \longrightarrow\{0,1\}$ como sendo a distância discreta sobre $S$, dada por

$$
c(x, y)= \begin{cases}1, & \text { se } x \neq y \\ 0, & \text { se } x=y\end{cases}
$$


Observe que para todo acoplamento $Q \in(\mu, \nu)$, vale que

$$
\iint_{S^{2}} c(x, y) Q(d x, d y)=\iint_{S_{1}} Q(d x, d y)=Q\left(S_{1}\right)
$$

onde $S_{1}=\left\{(x, y) \in S^{2}: c(x, y)=1\right\}=\{(x, y): x \neq y\}$. Logo, o mínimo de Monge-Kantorovich é dado, em termos de variáveis aleatórias, por

$$
K(\mu, \nu)=\inf _{X \sim \mu, Y \sim \nu} \mathbb{P}(X \neq Y),
$$

onde $X$ e $Y$ são duas variáveis aleatórias com leis $\mu$ e $\nu$, respectivamente.

Dobrushin [8, p. 62] estabeleceu a relação entre o mínimo de Monge-Kantorovich e a distância de variação total por meio do seguinte

Teorema 2.3.2 Sejam $(S, \mathcal{B})$ um espaço mensurável, $\mu, \nu \in \mathcal{P}(S)$ e c $(x, y)$ a distância discreta sobre o conjunto S. Então,

$$
K(\mu, \nu)=\|\mu-\nu\|_{V T}
$$

Este teorema é um dos diversos resultados relacionados com a teoria de acoplamentos maximais, que é a base para a demonstração via acoplamento do Teorema Limite para cadeias de Markov.

\subsection{O Teorema da Dualidade}

A Teoria da Dualidade para o problema do transporte ótimo começou quando Kantorovich demonstrou a equivalência de seu problema original (denominado problema primal) com um problema dual no caso em que $S$ é um espaço métrico compacto e $c(x, y)$ é uma função de custo contínua. Contudo, a demonstração deste fato valia somente no caso em que $c(x, y)$ é uma distância sobre $S$. Posteriormente, a teoria passou a ser estendida para espaços mais complexos e com funções de custo que não necessariamente eram distâncias. (Para mais informações, ver Rachev e Rüschendorf [37, Capítulo 2].)

Antes de apresentarmos o resultado principal desta seção, damos a seguinte

Definição 2.4.1 Dizemos que um espaço de probabilidade $(S, \mathcal{B}, \mu)$ é perfeito (ou, equivalentemente, $\mu$ é perfeita) se para toda função $\mathcal{B}$-mensurável $f: S \longrightarrow \mathbb{R}$ podemos obter um conjunto $B \subset f(S)$ tal que $\mu\left(f^{-1}(B)\right)=1$. 
O resultado básico da Teoria da Dualidade é uma extensão natural do Teorema de KantorovichRubinstein:

Teorema 2.4.2 (Teorema Geral da Dualidade.) Sejam $\mu, \nu \in \mathcal{P}(S)$ tais que $\mu$ é perfeita e uma função custo $c: S^{2} \longrightarrow \mathbb{R}_{+}$mensurável e tal que $c(x, y) \leq f_{1}(x)+f_{2}(y)$ para certas funçôes $f_{1} \in \mathcal{L}^{1}(\mu)$ e $f_{2} \in \mathcal{L}^{1}(\nu)$. Então, vale a seguinte dualidade:

$$
K(\mu, \nu)=\sup \left\{\int_{S} h_{1}(x) \mu(d x)+\int_{S} h_{2}(y) \nu(d y)\right\},
$$

onde o supremo é tomado sobre todas as funções $h_{1}, h_{2}: S \longrightarrow \mathbb{R}$ tais que

(i) $h_{1} \in \mathcal{L}^{1}(\mu)$ e $h_{2} \in \mathcal{L}^{1}(\nu)$;

(ii) $h_{1}(x)+h_{2}(y) \leq c(x, y)$ para quaisquer $x, y \in S$.

A interpretação do Teorema da Dualidade é a seguinte: sob certas restrições, resolver um problema de minimização de Monge-Kantorovich (problema primal) é equivalente a resolver um problema de maximização (problema dual). A partir desse resultado, iniciou-se um aprofundamento nos estudos da Teoria da Dualidade como ferramenta básica para a solução de diversos problemas, com destaque para problemas teóricos e práticos relacionados com a Programação Linear. 


\section{Capítulo 3}

\section{Construção de acoplamentos}

Neste capítulo, apresentamos uma condição necessária que um acoplamento de duas medidas de probabilidades definidas em um conjunto finito $S$ deve satisfazer para atingir o mínimo de MongeKantorovich - definido em (2.7) - quando a função custo assume valor nulo somente nos elementos da diagonal de $S^{2}$. Para todas as definições e resultados apresentados nas Seções 3.1 e 3.2 assumiremos que os elementos de $S$ podem ser enumerados como $1,2, \ldots, N$, onde $N$ é o número total de elementos de $S$.

Além disso, apresentamos na Seção 3.3 um resultado que está relacionado com a solução do problema de Monge-Kantorovich em espaços produto finitos.

\subsection{Acoplamentos e partições}

Dadas $\mu, \nu \in \mathcal{P}(S)$, definimos as seqüências $\mathcal{P}_{\mu}=\left\{I_{x}^{\mu}: x \in S\right\}$ e $\mathcal{P}_{\nu}=\left\{I_{y}^{\nu}: y \in S\right\}$ de subintervalos do intervalo $[0,1]$ que satisfaçam as seguintes propriedades:

(i) $\mathcal{P}_{\mu}$ e $\mathcal{P}_{\nu}$ formam duas partições do intervalo $[0,1]$, isto é, $I_{x}^{\mu} \cap I_{x^{\prime}}^{\mu}=\emptyset$ para $x \neq x^{\prime}, I_{y}^{\nu} \cap I_{y^{\prime}}^{\nu}=\emptyset$ para $y \neq y^{\prime}$ e

$$
\bigcup_{x \in S} I_{x}^{\mu}=\bigcup_{y \in S} I_{y}^{\nu}=[0,1]
$$

(ii) $\left|I_{x}^{\mu}\right|=\mu(x)$ para todo $x \in S$ e $\left|I_{y}^{\nu}\right|=\nu(y)$ para todo $y \in S$.

Seja $\mathcal{P}_{\mu, \nu}=\left\{I_{\mu, \nu}(x, y): x \in S\right.$ e $\left.y \in S\right\}$ uma outra partição de $[0,1]$, na qual cada elemento é da forma

$$
I_{\mu, \nu}(x, y):=I_{x}^{\mu} \cap I_{y}^{\nu}, \quad x, y \in S
$$


e, para esta partição, seja $Q$ a medida de probabilidade em $S^{2}$ definida por

$$
Q(x, y)=\left|I_{\mu, \nu}(x, y)\right|=\mathbb{P}\left(U \in I_{\mu, \nu}(x, y)\right), \quad(x, y) \in S^{2}
$$

em que $U$ é uma variável aleatória distribuída uniformemente em $[0,1]$.

Como conseqüência imediata da construção das partições $\mathcal{P}_{\nu}$ e $\mathcal{P}_{\nu}$, vale a seguinte

Proposição 3.1.1 A medida $Q$ é um acoplamento de $\mu$ e $\nu$, ou seja, $Q \in \mathcal{M}(\mu, \nu)$.

Demonstração. Basta observar que para cada $x \in S$ fixado, temos que

$$
\begin{aligned}
\sum_{y \in S} Q(x, y) & =\sum_{y \in S} \mathbb{P}\left(U \in I_{\mu, \nu}(x, y)\right)=\mathbb{P}\left(\bigcup_{y \in S}\left\{U \in I_{\mu, \nu}(x, y)\right\}\right) \\
& =\mathbb{P}\left(U \in \bigcup_{y \in S} I_{\mu, \nu}(x, y)\right)=\mathbb{P}\left(U \in I_{x}^{\mu}\right)=\mu(x)
\end{aligned}
$$

e

$$
\begin{aligned}
\sum_{x \in S} Q(x, y) & =\sum_{x \in S} \mathbb{P}\left(U \in I_{\mu, \nu}(x, y)\right)=\mathbb{P}\left(\bigcup_{x \in S}\left\{U \in I_{\mu, \nu}(x, y)\right\}\right) \\
& =\mathbb{P}\left(U \in \bigcup_{x \in S} I_{\mu, \nu}(x, y)\right)=\mathbb{P}\left(U \in I_{y}^{\nu}\right)=\nu(y)
\end{aligned}
$$

para cada $y \in S$ fixado. Isso completa a demonstração.

Até agora, descrevemos como construir uma partição que caracteriza o acoplamento $Q$. A próxima proposição garante a reciprocidade desta construção. Antes de o demonstrarmos, definimos a quantidade $\theta(Q)=\sum_{x \in S} Q(x, x)$.

Proposição 3.1.2 Dado $Q$ um acoplamento arbitrário de $\mu$ e $\nu$, é possível construir uma partição $\mathcal{P}_{Q}:=\left\{I_{Q}(x, y):(x, y) \in S^{2}\right\}$ do intervalo $[0,1]$ tal que

$$
\left|I_{Q}(x, y)\right|=Q(x, y), \quad(x, y) \in S^{2}
$$


Demonstração. Primeiramente, construímos os intervalos $\left\{I_{Q}(x, x): x \in S\right\}$ sobre o intervalo $[0,1]$ da seguinte forma:

$$
\begin{aligned}
I_{Q}(1,1) & =[0, Q(1,1)[ \\
I_{Q}(x, x) & =\left[\sum_{z=1}^{x-1} Q(z, z), \sum_{z=1}^{x} Q(z, z)[\text { para } x=2, \ldots, N-1\right. \\
I_{Q}(N, N) & =\left[\sum_{z=1}^{N-1} Q(z, z), \theta(Q)[\right.
\end{aligned}
$$

Observe que, de fato, $\left|I_{Q}(x, x)\right|=Q(x, x)$ para todo $x \in S$.

Os intervalos restantes podem ser construídos de forma análoga no subintervalo $[\theta(Q), 1]$, de modo que satisfaçam (3.3). Como exemplo, podemos definir inicialmente

$$
\begin{array}{rcc}
I_{Q}(1,2)= & {[\theta(Q), \theta(Q)+Q(1,2)[} \\
I_{Q}(1,3)= & {[\theta(Q)+Q(1,2), \theta(Q)+Q(1,2)+Q(1,3)[} \\
\vdots & \vdots \\
I_{Q}(1, N)= & {\left[\theta(Q)+\sum_{y=2}^{N-1} Q(1, y), \theta(Q)+\sum_{y=2}^{N} Q(1, y)[\right.}
\end{array}
$$

e construir os intervalos restantes da mesma forma, o que completa a demonstração.

A seguir, apresentamos uma desigualdade que será a base para a formulação e demonstração do teorema da Seção 3.2:

Proposição 3.1.3 (Desigualdade de Fréchet) Para todo $x \in S$ e para todo $y \in S$, temos que

$$
\max \{\mu(x)+\nu(y)-1,0\} \leq Q(x, y) \leq \min \{\mu(x), \nu(y)\}
$$

Demonstração. O limite superior da desigualdade é imediato, uma vez que dado um acoplamento arbitrário $Q$, segue que $\mu(x) \geq Q(x, y)$ e $\nu(y) \geq Q(x, y)$ pela definição de $Q$. 
Para o limite inferior, seja $(X, Y)$ com distribuição conjunta dada por $Q$. Então, pela Teoria das Probabilidades, segue que

$$
\begin{aligned}
1 & \geq \mathbb{P}(\{X=x\} \cup\{Y=y\})=\mathbb{P}(X=x)+\mathbb{P}(Y=y)-\mathbb{P}(\{X=x\} \cap\{Y=y\}) \\
& =\mu(x)+\nu(y)-Q(x, y) \Leftrightarrow Q(x, y) \geq \mu(x)+\nu(y)-1 .
\end{aligned}
$$

E como $Q(x, y) \geq 0$, o limite inferior da desigualdade está verificado, o que completa a demonstração.

Finalmente, dado o acoplamento $Q$, definimos duas partições do intervalo $[\theta(Q), 1]$ denotadas por $\mathcal{R}_{\mu}=\left\{R_{x}^{\mu}: x \in S\right\}$ e $\mathcal{R}_{\nu}=\left\{R_{y}^{\nu}: y \in S\right\}$ tais que

$$
R_{x}^{\mu}=\bigcup_{\substack{y \in S \\ y \neq x}} I_{Q}(x, y) \text { e } R_{y}^{\nu}=\bigcup_{\substack{x \in S \\ x \neq y}} I_{Q}(x, y)
$$

onde $\left\{I_{Q}(x, y):(x, y) \in S^{2}\right\}$ é uma partição que caracteriza $Q$ e é tal que

$$
\bigcup_{x \in S} I_{Q}(x, x)=[0, \theta(Q)[
$$

Observe também que

$$
\left|R_{x}^{\mu}\right|=\mu(x)-Q(x, x) \text { e }\left|R_{y}^{\nu}\right|=\nu(y)-Q(y, y)
$$

para quaisquer $x, y \in S$. Por este motivo, os intervalos pertencentes a $\mathcal{R}_{\mu}$ e $\mathcal{R}_{\nu}$ serão denominados restos em relação a $\mu$ e $\nu$, respectivamente.

\subsection{Uma condição necessária}

Seja $c: S^{2} \longrightarrow \mathbb{R}_{+}$uma função real tal que:

- $c(x, y) \geq 0$, para todo $(x, y) \in S^{2}$

- $c(x, y)=0$ se e somente se $x=y$.

Nosso objetivo é construir um acoplamento $\bar{Q} \in \mathcal{M}(\mu, \nu)$ que atinge o mínimo de Monge-Kantorovich. O teorema presente nesta seção, juntamente com seu respectivo corolário, apresenta uma condição 
necessária para que tal mínimo seja alcançado. Em primeiro lugar, enunciamos o teorema a seguir:

Teorema 3.2.1 Dado $Q \in \mathcal{M}(\mu, \nu)$ um acoplamento arbitrário tal que $\theta(Q)<\sum_{x \in S} \min \{\mu(x), \nu(x)\}$, então existe um outro acoplamento $\bar{Q} \in \mathcal{M}(\mu, \nu)$, com $\theta(\bar{Q})>\theta(Q)$, tal que

$$
\sum_{(x, y) \in S^{2}} c(x, y) \bar{Q}(x, y)<\sum_{(x, y) \in S^{2}} c(x, y) Q(x, y)
$$

A idéia por trás do Teorema 3.2.1 está ligada ao limite superior da Proposição 3.1.3, apresentada na seção anterior. Se $c$ possui valor nulo para os elementos da diagonal de $S^{2}$, é razoável imaginar que o acoplamento $Q^{*}$ que atinge o mínimo de Monge-Kantorovich deveria ter probabilidade máxima na diagonal de $S^{2}$, ou seja, $Q(x, x)=\min \{\mu(x), \nu(x)\}$ para todo $x \in S$. O seguinte corolário do Teorema 3.2.1 garante que tal raciocínio é perfeitamente aceitável:

Corolário 3.2.2 Uma condição necessária para que um acoplamento $\bar{Q} \in \mathcal{M}(\mu, \nu)$ atinga o mínimo de Monge-Kantorovich é que $\bar{Q}(x, x)=\min \{\mu(x), \nu(x)\}$, para todo $x \in S$.

Antes da demonstração do teorema e de seu Corolário, apresentamos dois lemas que serão fundamentais na demonstração dos mesmos, uma vez que estão diretamente relacionados com a construção do acoplamento $Q$ sobre o intervalo $[0,1]$.

Lema 3.2.3 $\theta(Q)<\sum_{x \in S} \min \{\mu(x), \nu(x)\} \Leftrightarrow\left\{x \in S:\left|R_{x}^{\mu}\right|>0\right\} \cap\left\{x \in S:\left|R_{x}^{\nu}\right|>0\right\} \neq \emptyset$

\section{Demonstração.}

$(\Rightarrow)$ Se $\theta(Q)<\sum_{x \in S} \min \{\mu(x), \nu(x)\}$, então existe pelo menos um $\bar{x} \in S$ tal que a desigualdade $Q(\bar{x}, \bar{x})<\min \{\mu(\bar{x}), \nu(\bar{x})\}$ vale, de modo que $\left|R_{\bar{x}}^{\mu}\right|=\mu(\bar{x})-Q(\bar{x}, \bar{x})>0$ e $\left|R_{\bar{x}}^{\nu}\right|=\nu(\bar{x})-Q(\bar{x}, \bar{x})>0$.

$(\Leftarrow)$ Analogamente, se $\left\{x \in S:\left|R_{x}^{\mu}\right|>0\right\} \cap\left\{x \in S:\left|R_{x}^{\nu}\right|>0\right\} \neq \emptyset$, então existe pelo menos um $\bar{x} \in S$ tal que $\left|R_{\bar{x}}^{\mu}\right|=\mu(\bar{x})-Q(\bar{x}, \bar{x})>0$ e $\left|R_{\bar{x}}^{\nu}\right|=\nu(\bar{x})-Q(\bar{x}, \bar{x})>0$ e, portanto, temos que $Q(\bar{x}, \bar{x})<\min \{\mu(\bar{x}), \nu(\bar{x})\}$, o que completa a demonstração do lema. 
Lema 3.2.4 Se $\mathcal{E}=\left\{x \in S:\left|R_{x}^{\mu}\right|>0\right\} \cap\left\{x \in S:\left|R_{x}^{\nu}\right|>0\right\} \neq \emptyset$, então existe um reordenamento dos intervalos $\left\{R_{x}^{\mu}:\left|R_{x}^{\mu}\right|>0, x \in S\right\}$ e $\left\{R_{x}^{\nu}:\left|R_{x}^{\nu}\right|>0, x \in S\right\}$ de modo que, neste novo reordenamento, temos que

$$
\tilde{R}_{\bar{x}}^{\mu} \subset \tilde{R}_{\bar{x}}^{\nu} \text { ou } \tilde{R}_{\bar{x}}^{\nu} \subset \tilde{R}_{\bar{x}}^{\mu}
$$

para algum $\bar{x} \in \mathcal{S}$.

Demonstração. De fato, se $\mathcal{E} \neq \emptyset$, então existe pelo menos um $\bar{x} \in S$ tal que $\left|R_{\bar{x}}^{\mu}\right|>0$ e $\left|R_{\bar{x}}^{\nu}\right|>0$ e, por conseqüência, $\mu(\bar{x})>0$ e $\nu(\bar{x})>0$. Para este $\bar{x}$, assumiremos que $\left|R_{\bar{x}}^{\mu}\right| \leq\left|R_{\bar{x}}^{\nu}\right|$.

Construímos duas novas partições de restos $\tilde{\mathcal{R}}_{\mu}=\left\{\tilde{R}_{x}^{\mu}: x \in S\right\}$ e $\tilde{\mathcal{R}}_{\nu}=\left\{\tilde{R}_{y}^{\nu}: y \in S\right\}$ de tal forma que

$$
\begin{aligned}
\left|\tilde{R}_{x}^{\mu}\right| & =\left|R_{x}^{\mu}\right|, \text { para todo } x \in S \backslash\{\bar{x}\} \\
\left|\tilde{R}_{y}^{\nu}\right| & =\left|R_{y}^{\nu}\right|, \text { para todo } y \in S \backslash\{\bar{x}\} \\
\tilde{R}_{\bar{x}}^{\mu} & =\left[\theta(Q), \theta(Q)+\left|R_{\bar{x}}^{\mu}\right|\right] \\
\tilde{R}_{\bar{x}}^{\nu} & =\left[\theta(Q), \theta(Q)+\left|R_{\bar{x}}^{\nu}\right|\right] .
\end{aligned}
$$

Nessas condições, é imediato que $\tilde{R}_{\bar{x}}^{\mu} \subset \tilde{R}_{\bar{x}}^{\nu}$. Como a construção dos restos é igual quando $\left|R_{\bar{x}}^{\nu}\right| \leq\left|R_{\bar{x}}^{\mu}\right|$, a demonstração do lema está completa.

Finalmente, apresentamos as demonstrações do Teorema 3.2.1 e do Corolário 3.2.2.

Demonstração do Teorema 3.2.1. Seja $\mathcal{P}_{Q}=\left\{I_{Q}(x, y):(x, y) \in S^{2}\right\}$ uma partição de $[0,1]$ que determina o acoplamento $Q$ como na Proposição 3.1.2. Pelos Lemas 3.2.3 e 3.2.4, existe um $\bar{x} \in S$ tal que podemos construir duas partições de restos $\tilde{\mathcal{R}}_{\mu}$ e $\tilde{\mathcal{R}}_{\nu}$ como em (3.8) de modo que $\tilde{R}_{\bar{x}}^{\mu} \subset \tilde{R}_{\bar{x}}^{\nu}$ ou $\tilde{R}_{\bar{x}}^{\nu} \subset \tilde{R}_{\bar{x}}^{\mu}$.

Assumiremos que $\tilde{R}_{\bar{x}}^{\mu} \subset \tilde{R}_{\bar{x}}^{\nu}$. Isso implica que

$$
\left|\tilde{R}_{\bar{x}}^{\mu}\right|<\left|\tilde{R}_{\bar{x}}^{\nu}\right| \Rightarrow \mu(\bar{x})-Q(\bar{x}, \bar{x})<\nu(\bar{x})-Q(\bar{x}, \bar{x}) \Rightarrow \mu(\bar{x})<\nu(\bar{x})
$$

e, portanto, $\min \{\mu(\bar{x}), \nu(\bar{x})\}=\mu(\bar{x})>0$. 
Construímos uma nova partição $\overline{\mathcal{P}}=\left\{\bar{I}(x, y):(x, y) \in S^{2}\right\}$ de tal forma que

$$
\begin{cases}|\bar{I}(u, u)|=\left|I_{Q}(u, u)\right|, & \text { se } x \in S \backslash\{\bar{x}\} \\ |\bar{I}(\bar{x}, \bar{x})|=\left|I_{Q}(\bar{x}, \bar{x})\right|+\left|\tilde{R}_{\bar{x}}^{\mu}\right|=\mu(\bar{x}), & \end{cases}
$$

e os respectivos restos são definidos de tal forma que

$$
\begin{cases}\left|\bar{R}_{x}^{\mu}\right|=\left|\tilde{R}_{x}^{\mu}\right| \text { e }\left|\bar{R}_{x}^{\nu}\right|=\left|\tilde{R}_{x}^{\nu}\right|, & \text { se } x \in S \backslash\{\bar{x}\} \\ \left|\bar{R}_{\bar{x}}^{\mu}\right|=\emptyset \text { e }\left|\bar{R}_{\bar{x}}^{\nu}\right|=\left|\tilde{R}_{\bar{x}}^{\nu}\right|-\left|\tilde{R}_{\bar{x}}^{\mu}\right|=\nu(\bar{x})-\mu(\bar{x}) . & \end{cases}
$$

Definimos o acoplamento $\bar{Q}$ como

$$
\bar{Q}(x, y)=\mathbb{P}(U \in \bar{I}(x, y)), \quad(x, y) \in S^{2}
$$

Da construção de $\bar{Q}$, segue imediatamente que

$$
\theta(\bar{Q})=\sum_{x \in S \backslash\{\bar{x}\}} \bar{Q}(x, x)+\bar{Q}(\bar{x}, \bar{x})>\sum_{x \in S \backslash\{\bar{x}\}} Q(x, x)+Q(\bar{x}, \bar{x})=\theta(Q)
$$

e, portanto,

$$
\begin{aligned}
\sum_{(x, y) \in S^{2}} c(x, y) \bar{Q}(x, y) & =\sum_{(x, y) \in S^{2}: x \neq y} c(x, y) \bar{Q}(x, y) \\
& <\sum_{(x, y) \in S^{2}: x \neq y} c(x, y) Q(x, y)=\sum_{(x, y) \in S^{2}} c(x, y) Q(x, y)
\end{aligned}
$$

e, com isso, a demonstração do teorema está completa.

Demonstração do Corolário 3.2.2. A demonstração é imediata, uma vez que basta observar que, dado qualquer acoplamento $Q$ tal que $\theta(Q)<\sum_{x \in S} \min \{\mu(x), \nu(x)\}$, sempre é possível construir um outro acoplamento definido como em (3.9) e seja tal que (3.10) vale e o custo esperado diminui. Logo, é necessário que $\theta(\bar{Q})=\sum_{x \in S} \min \{\mu(x), \nu(x)\}$, o que completa a demonstração do corolário. 


\subsection{Acoplamentos em espaços produto}

Seja $S_{1}, \ldots, S_{n}$ uma seqüência finita de conjuntos finitos. Para cada $i=1, \ldots, n$, sejam $\mu_{i}$ e $\nu_{i}$ duas medidas de probabilidade sobre $S_{i}$. Definimos $S$ como o espaço produto

$$
S=S_{1} \times \ldots \times S_{n}
$$

e $\mu$ e $\nu$ as seguintes medidas produto sobre $S$ :

$$
\begin{array}{r}
\mu=\mu_{1} \times \ldots \times \mu_{n}, \\
\nu=\nu_{1} \times \ldots \times \nu_{n} .
\end{array}
$$

Definimos também $\mathcal{M}^{P}(\mu, \nu)$ como sendo o conjunto dos acoplamentos entre $\mu$ e $\nu$ que são medidas produto. Observe que $\mathcal{M}^{P}(\mu, \nu) \subset \mathcal{M}(\mu, \nu)$, uma vez que nem todo acoplamento $Q$ de $\mu$ e $\nu$ é medida produto $^{1}$.

Se $x=\left(x_{1}, \ldots, x_{n}\right)$ é um elemento de $S$, para cada $i=1, \ldots, n$, definimos $x_{i}$ como a $i$-ésima coordenada de $x$. Dado um acoplamento $Q \in \mathcal{M}(\mu, \nu)$, definimos $Q_{i}$ como sendo a projeção de $Q$ sobre $S_{i}$. Em outras palavras, para qualquer par ordenado $\left(x_{i}, y_{i}\right)$ de elementos de $S_{i}$, definimos

$$
Q_{i}\left(x_{i}, y_{i}\right)=\sum_{(u, v) \in S^{2}:\left(u_{i}, v_{i}\right)=\left(x_{i}, y_{i}\right)} Q(u, v)
$$

Observe que $Q \in \mathcal{M}^{P}(\mu, \nu)$ se e somente se

$$
Q=Q_{1} \times \ldots \times Q_{n}
$$

Para cada $i=1, \ldots, n$ introduzimos as funções custo $c_{i}: S_{i}^{2} \longrightarrow \mathbb{R}_{+}$e, a partir delas, definimos uma função custo $c$ sobre $S$ da seguinte forma:

$$
c(x, y)=\sum_{i=1}^{n} c_{i}\left(x_{i}, y_{i}\right), \quad x, y \in S .
$$

\footnotetext{
${ }^{1} \mathrm{O}$ Exemplo 1 da Seção 4.1 apresenta dois acoplamentos que não são medidas produto, uma vez que a relação $Q(A, B)=\mu(A) \nu(B)$ não está satisfeita para quaisquer $A$ e $B$ subconjuntos de $S$.
} 
Finalmente, introduzimos a notação

$$
\mathbb{E}_{Q}(c)=\sum_{(x, y) \in S^{2}} Q(x, y) c(x, y)
$$

Enunciamos o resultado principal desta Seção:

Teorema 3.3.1 Se para cada $i=1, \ldots, n$ o acoplamento $P_{i}^{*} \in \mathcal{M}\left(\mu_{i}, \nu_{i}\right)$ é uma solução do problema de Monge-Kantorovich com respeito a $\mu_{i}, \nu_{i}$ e $c_{i}$, então a medida produto

$$
Q^{*}=P_{1}^{*} \times \ldots \times P_{n}^{*}
$$

é uma solução do problema de Monge-Kantorovich com respeito a $\mu, \nu$ e c.

A demonstração deste teorema segue a partir de dois lemas, que são enunciados e demonstrados a seguir.

Lema 3.3.2 Para todo acoplamento $Q \in \mathcal{M}(\mu, \nu)$, temos que

$$
\mathbb{E}_{Q}(c)=\sum_{i=1}^{n} \mathbb{E}_{Q_{i}}\left(c_{i}\right)
$$

Demonstração. Em primeiro lugar, observe que

$$
\mathbb{E}_{Q}(c)=\sum_{(x, y) \in S^{2}} Q(x, y)\left[\sum_{i=1}^{n} c_{i}\left(x_{i}, y_{i}\right)\right]=\sum_{i=1}^{n} \sum_{(x, y) \in S^{2}} Q(x, y) c_{i}\left(x_{i}, y_{i}\right) .
$$

Para cada $i$ fixado, temos que

$$
\sum_{(x, y) \in S^{2}} Q(x, y) c_{i}\left(x_{i}, y_{i}\right)=\sum_{\left(x_{i}, y_{i}\right) \in S_{i}^{2}} \sum_{(u, v) \in S^{2}:\left(u_{i}, v_{i}\right)=\left(x_{i}, y_{i}\right)} Q(u, v) c_{i}\left(x_{i}, y_{i}\right)
$$

O lado direito da equação é, por definição, igual a

$$
\sum_{\left(x_{i}, y_{i}\right) \in S_{i}^{2}} Q_{i}\left(x_{i}, y_{i}\right) c_{i}\left(x_{i}, y_{i}\right)=\mathbb{E}_{Q_{i}}\left(c_{i}\right)
$$


o que conclui a demonstração do lema.

Lema 3.3.3 Para todo $Q \in \mathcal{M}(\mu, \nu)$, temos que

$$
\mathbb{E}_{Q_{i}}\left(c_{i}\right) \geq \mathbb{E}_{P_{i}^{*}}\left(c_{i}\right)
$$

Demonstração. Em primeiro lugar, observamos que para $x_{i}$ fixado, temos que

$$
\sum_{y_{i} \in S_{i}} Q_{i}\left(x_{i}, y_{i}\right)=\sum_{u \in S: u_{i}=x_{i}} \sum_{y \in S} Q(u, y)
$$

O lado direito da equação é igual a

$$
\sum_{u \in S: u_{i}=x_{i}} \mu(u)=\mu_{i}\left(x_{i}\right)
$$

Analogamente, para cada $y_{i}$ fixado, temos que

$$
\sum_{x_{i} \in S_{i}} Q_{i}\left(x_{i}, y_{i}\right)=\nu_{i}\left(y_{i}\right)
$$

Portanto, $Q_{i}$ é um acoplamento de $\mu_{i}$ e $\nu_{i}$. Como, por hipótese, $P_{i}^{*}$ é uma solução do problema de Monge-Kantorovich com respeito a $\mu_{i}, \nu_{i}$ and $c_{i}$, a desigualdade proposta é válida, concluindo a demonstração do lema.

Demonstração do Teorema 3.3.1. A partir dos dois lemas, a demonstração é imediata. Pelo Lema 3.3.2, minimizar $\mathbb{E}_{Q}(c)$ equivale a minimizar $\sum_{i=1}^{n} \mathbb{E}_{Q_{i}}\left(c_{i}\right)$. Uma vez que cada termo da soma é não-negativo, é suficiente minimizar cada uma das esperanças $\mathbb{E}_{Q_{i}}\left(c_{i}\right)$.

Portanto, qualquer medida que minimize $\mathbb{E}_{Q_{i}}\left(c_{i}\right)$ para cada $i=1, \ldots, n$ é uma solução do problema de Monge-Kantorovich com respeito a $\mu, \nu$ e $c$.

Pelo Lema 3.3.3, isso implica que as marginais $Q_{i}$, para $i=1, \ldots, n$, devem satisfazer as equações

$$
\mathbb{E}_{Q_{i}}\left(c_{i}\right)=\mathbb{E}_{P_{i}^{*}}\left(c_{i}\right)
$$


A medida produto

$$
Q^{*}=P_{1}^{*} \times \ldots \times P_{n}^{*}
$$

possui esta propriedade, uma vez que para cada $i=1, \ldots, n$, temos que $Q_{i}^{*}=P_{i}^{*}$. Isso conclui a demonstração do teorema.

Como conseqüência, deduzimos que sob as hipóteses do Teorema 3.3.1, para resolver o problema de Monge-Kantorovich com relação a $\mu, \nu$ e $c$, é suficiente procurar por um acoplamento ótimo em um conjunto muito menor - o conjunto de acoplamentos construídos como medidas produto:

Corolário 3.3.4 Sob as hipóteses do Teorema 3.3.1, temos que

$$
\inf _{Q \in \mathcal{M}(\mu, \nu)} \mathbb{E}_{Q}(c)=\inf _{Q \in \mathcal{M}^{P}(\mu, \nu)} \mathbb{E}_{Q}(c)
$$




\section{Capítulo 4}

\section{Exemplos}

Neste capítulo, apresentamos exemplos nos quais pode-se obter o mínimo de Monge-Kantorovich explicitamente. Os exemplos relativos ao caso contínuo do problema de Monge-Kantorovich são resultados conhecidos e que são encontrados na literatura sobre o assunto.

\subsection{Exemplos no caso finito}

Exemplo 1. Consideraremos o caso finito do problema de Monge-Kantorovich, com $S=\{1,2, \ldots, N\}$ e $c(x, y)$ a distância discreta definida em (2.15). O mínimo de Monge-Kantorovich é dado por

$$
K(\mu, \nu)=\min \left\{\mathbb{E}_{Q}(c): Q \in \mathcal{M}(\mu, \nu)\right\}
$$

onde $\mathbb{E}_{Q}(c)$ é o valor esperado de $c$ em relação ao acoplamento $Q$, como definido em (3.15):

$$
\mathbb{E}_{Q}(c)=\sum_{(x, y) \in S^{2}} c(x, y) Q(x, y)=\sum_{(x, y) \in S^{2}: x \neq y} Q(x, y)=1-\sum_{x \in S} Q(x, x)=1-\theta(Q) .
$$

Nestas condições, a suficiência do Corolário 3.2.2 é válida, uma vez que se um acoplamento $\bar{Q}$ atinge $K(\mu, \nu)$, então $\theta(\bar{Q})$ deve ser máximo, o que ocorre quando $\bar{Q}(x, x)$ atinge o limite superior da desigualdade de Fréchet para todo $x \in S$, ou seja,

$$
\bar{Q}(x, x)=\min \{\mu(x), \nu(x)\}, \quad \forall x \in S,
$$

e, portanto, $\bar{Q}$ é uma solução ótima do problema de Monge-Kantorovich. Entretanto, não há garantias quanto à unicidade desta solução ótima. Como exemplo, tomemos $S=\{1,2,3,4\}$ e $\mu, \nu$ definidas da 
seguinte forma:

$$
\left\{\begin{array}{l}
\mu(1)=\nu(4)=0.1 \\
\mu(2)=\nu(3)=0.2 \\
\mu(3)=\nu(2)=0.3 \\
\mu(4)=\nu(1)=0.4
\end{array}\right.
$$

Agora, definimos dois acoplamentos $Q_{1}$ e $Q_{2}$ de $\mu$ e $\nu$ da seguinte forma:

$$
Q_{1}:\left\{\begin{array}{l}
Q_{1}(1,1)=Q_{1}(4,4)=0.1 \\
Q_{1}(2,2)=Q_{1}(3,3)=0.2 \\
Q_{1}(3,1)=Q_{1}(4,2)=0.1 \\
Q_{1}(4,1)=0.2 \\
Q_{1}(x, y)=0 \quad \text { para os demais pontos }(x, y) \in S^{2}
\end{array}\right.
$$

e

$$
Q_{2}:\left\{\begin{array}{l}
Q_{2}(1,1)=Q_{2}(4,4)=0.1 \\
Q_{2}(2,2)=Q_{2}(3,3)=0.2 \\
Q_{2}(3,2)=0.1 \\
Q_{2}(4,1)=0.3 \\
Q_{2}(x, y)=0 \quad \text { para os demais pontos }(x, y) \in S^{2}
\end{array}\right.
$$

Observe que $Q_{1}$ e $Q_{2}$ são dois acoplamentos diferentes. Entretanto, observe também que para cada $i$ fixado, $i=1,2$, temos que $Q_{i}(x, x)=\min \{\mu(x), \nu(x)\}$ para todo $x \in S$. Logo, $Q_{1}$ e $Q_{2}$ são soluções ótimas do problema de Monge-Kantorovich, com

$$
K(\mu, \nu)=\mathbb{E}_{Q_{1}}(c)=\mathbb{E}_{Q_{2}}(c)=1-0.6=0.4 .
$$

Exemplo 2. Sejam $S=\{1, \ldots, N\}$ e $c(x, y)$ uma função custo não-negativa tal que

$$
c(x, y)=c(|x-y|), \quad \text { para todo } x, y \in S,
$$


com $0=c(0)<c(1)<\ldots<c(N-1)$. Tomemos $\mu, \nu \in \mathcal{P}(S)$ tais que $\mu(x) \leq \nu(x)$ para todo $x=1, \ldots, N-1$ e $\mu(N)>\nu(N)$.

Vamos construir um acoplamento $Q^{*}$ que atinge o mínimo de Monge-Kantorovich. Em primeiro lugar, de acordo com o Corolário 3.2.2, definimos

$$
Q^{*}(x, x)=\min \{\mu(x), \nu(x)\}, \quad \text { para todo } x \in S,
$$

Logo, vale que

$$
Q^{*}(x, x)=\left\{\begin{array}{l}
\mu(x), \text { se } x=1, \ldots, N-1 \\
\nu(x), \text { se } x=N
\end{array}\right.
$$

De (4.4) e da definição de acoplamento, segue que para todo $x=1, \ldots, N-1$ fixado, vale que

$$
Q^{*}(x, y)=0 \quad \text { para todo } y \neq x
$$

Resta determinar as probabilidades (em termos de $Q^{*}$ ) dos pontos da forma $(N, y), y=1, \ldots, N-1$. Contudo, podemos determinar essas probabilidades diretamente; basta observar que, para todo $y=$ $1, \ldots N-1$ fixado, vale que

$$
\nu(y)=\sum_{z=1}^{N} Q^{*}(z, y)=\sum_{z=1}^{N-1} Q^{*}(z, y)+Q^{*}(N, y)=Q^{*}(y, y)+Q^{*}(N, y)=\mu(y)+Q^{*}(N, y),
$$

uma vez que $Q^{*}(y, y)=\mu(y)$ e $Q^{*}(z, y)=0$ para todo $z \notin\{y, N\}$. Portanto, $Q^{*}(N, y)=\nu(y)-\mu(y)$ para todo $y=1, \ldots, N-1$.

Finalmente, como $Q^{*}$ é o único acoplamento que pode ser construído a partir do Corolário 3.2.2, segue que ele atinge o mínimo de Monge-Kantorovich, ou seja,

$$
K(\mu, \nu)=\mathbb{E}_{Q^{*}}(c)
$$

\subsection{Exemplos no caso contínuo}

Exemplo 1. (Dobrushin [8, p. 60]) Seja $S=\mathbb{R}^{n}$ com a distância Euclidiana

$$
c(x, y)=|x-y|
$$


Consideramos agora $\mu, \nu \in \mathcal{P}\left(\mathbb{R}^{n}\right)$ tais que, para algum vetor $a \in \mathbb{R}^{n}$ conhecido,

$$
\nu(B)=\mu(B-a), \quad B \in \mathcal{B}\left(\mathbb{R}^{n}\right) .
$$

Observe que se $\bar{Q} \in \mathcal{M}(\mu, \nu)$ é um acoplamento que está concentrado na diagonal

$$
\bar{Q}(\{(x, y): y=x+a\})=1
$$

então

$$
\iint_{S \times S}|x-y| \bar{Q}(d x, d y)=|a| .
$$

Por outro lado, para qualquer $Q \in \mathcal{M}(\mu, \nu)$, vale que

$$
\begin{aligned}
\iint_{S \times S}|x-y| Q(d x, d y) & \geq\left|\iint_{S \times S} x Q(d x, d y)-\iint_{S \times S} y Q(d x, d y)\right| \\
& =\left|\int_{S} x \mu(d x)-\int_{S} y \nu(d y)\right|=|a| .
\end{aligned}
$$

De (4.10) e (4.11), segue que

$$
K(\mu, \nu)=|a|
$$

Exemplo 2. Sejam $S=\mathbb{R}, c(x, y)$ definida como em (4.8) e $F_{1}, F_{2}$ as funções de distribuição acumuladas de $\mu, \nu \in \mathcal{P}(\mathbb{R})$, respectivamente:

$$
F_{1}\left(x_{0}\right)=\mu\left(\left\{x \in \mathbb{R}: x \leq x_{0}\right\}\right) \text { e } F_{2}\left(x_{0}\right)=\nu\left(\left\{x \in \mathbb{R}: x \leq x_{0}\right\}\right), \quad x_{0} \in \mathbb{R} .
$$

Nestas condições, Rachev [34, p. 653] demonstrou que o mínimo de Monge-Kantorovich é dado por

$$
K(\mu, \nu)=\int_{-\infty}^{\infty}\left|F_{1}(x)-F_{2}(x)\right| d x
$$

A demonstração deste resultado segue também a partir do seguinte teorema apresentado por Rachev e Rüschendorf [37, p. 17]: 
Teorema 4.2.1 Assuma que $c(x, y)=|x-y| \lambda(x, y)$, com $x, y \in \mathbb{R}$, e que para $x<t<y$, vale que $\lambda(t, t) \leq \lambda(x, y)$. Assuma também que $\lambda(x, y)$ é simétrica, contínua na diagonal e $t \rightarrow \lambda(t, t)$ é localmente limitada. Então,

$$
K(\mu, \nu)=\int_{-\infty}^{\infty} \lambda(t, t)\left|F_{1}(t)-F_{2}(t)\right| d t
$$

De fato, a igualdade (4.14) segue imediatamente quando tomamos $\lambda(x, y)=1$ para todo $x, y \in \mathbb{R} \mathrm{e}$ substituímos em (4.15).

Exemplo 3. Sejam $S=\mathbb{R}^{p}, c(\mathbf{x}, \mathbf{y})=|\mathbf{x}-\mathbf{y}|^{2}$ e $\mathbf{X}$ e $\mathbf{Y}$ dois vetores aleatórios em $\mathbb{R}^{p}$ com distribuições $N_{p}\left(\mathbf{m}_{\mathbf{1}}, \boldsymbol{\Sigma}_{\mathbf{1}}\right)$ e $N_{p}\left(\mathbf{m}_{\mathbf{2}}, \boldsymbol{\Sigma}_{\mathbf{2}}\right)$, respectivamente. As medidas de probabilidade associadas a $\mathbf{X}$ e $\mathbf{Y}$ são, respectivamente,

$$
\mu(A)=\int_{A} \frac{1}{\left(2 \pi\left|\mathbf{\Sigma}_{\mathbf{1}}\right|\right)^{p / 2}} \exp \left\{-\left(\mathbf{x}-\mathbf{m}_{\mathbf{1}}\right)^{T} \mathbf{\Sigma}_{\mathbf{1}}^{-\mathbf{1}}\left(\mathbf{x}-\mathbf{m}_{\mathbf{1}}\right)\right\} d \mathbf{x}, \quad A \in \mathcal{B}\left(\mathbb{R}^{p}\right),
$$

e

$$
\nu(B)=\int_{B} \frac{1}{\left(2 \pi\left|\boldsymbol{\Sigma}_{\mathbf{2}}\right|\right)^{p / 2}} \exp \left\{-\left(\mathbf{y}-\mathbf{m}_{\mathbf{2}}\right)^{T} \mathbf{\Sigma}_{\mathbf{2}}^{-\mathbf{1}}\left(\mathbf{y}-\mathbf{m}_{\mathbf{2}}\right)\right\} d \mathbf{y}, B \in \mathcal{B}\left(\mathbb{R}^{p}\right) .
$$

Nessas condições, Dowson e Landau [9] demonstraram o seguinte

Teorema 4.2.2 Dadas $\mu$ e $\nu$ como em (4.16) e (4.17), o minimo de Monge-Kantorovich satisfaz a igualdade

$$
[K(\mu, \nu)]^{2}=\left|\mathbf{m}_{\mathbf{1}}-\mathbf{m}_{\mathbf{2}}\right|^{2}+\operatorname{tr}\left[\boldsymbol{\Sigma}_{\mathbf{1}}+\boldsymbol{\Sigma}_{\mathbf{2}}-2\left(\boldsymbol{\Sigma}_{\mathbf{1}} \boldsymbol{\Sigma}_{\mathbf{2}}\right)^{\frac{1}{2}}\right]
$$




\section{Capítulo 5}

\section{Programação Linear}

A Programação Linear surgiu a partir da necessidade da construção de um modelo que tivesse aplicação em uma grande classe de problemas de decisão existentes em diversas áreas, como Indústria, Economia, Logística e Engenharia. Sua teoria engloba uma das estruturas matemáticas mais simples que pode ser usada para resolver problemas práticos de planejamento associados com essas áreas. Neste capítulo, discutimos a relação da teoria de Programação Linear com o problema de MongeKantorovich.

\section{$5.1 \quad$ Origens}

A Programação Linear ${ }^{1}$ está ligada com a descrição das relações entre componentes de um sistema. Em termos matemáticos, definimos um problema de Programação Linear (ou simplesmente problema linear) como o problema de minimizar (ou maximizar) uma função linear (denominada função objetivo) sujeito a certas restrições, que são determinadas por meio de um sistema formado por equações e/ou inequações lineares.

Em termos de teoria matemática, os primeiros passos da Programação Linear são atribuídos a C.F. Gauss e W. Jordan que, no início do Século XIX, desenvolveram métodos para a resolução de sistemas de equações lineares. Outro matemático importante e que foi contemporâneo de Gauss e Jordan, J. Fourier foi um dos primeiros a propor um problema de minimização sujeito a restrições lineares [13].

\footnotetext{
${ }^{1}$ T.C. Koopmans sugeriu o termo "Programação Linear" pela primeira vez em 1951 como uma alternativa à forma anterior, "Programação sob uma Estrutura Linear" (Dantzig [5, p. 6]).
} 
A teoria matemática da Programação Linear desenvolveu-se rapidamente e de forma mais intensa durante a Segunda Guerra Mundial (1939-1945), com aplicações diretas na logística dos Exércitos (em especial, o Exército norte-americano) que combateram durante o período. Uma dessas aplicações foi a distribuição de alimentos e suprimento de guerra, que deveria seguir critérios como proporcionalidade e aditividade (critérios utilizados na construção de um modelo de Programação Linear) com o menor custo possível. Dentre os principais trabalhos do período, destacam-se o primeiro trabalho de L.V. Kantorovich em 1939 [21], a forma padrão do problema do transporte, proposta por F.L. Hitchcock em 1941 [17] e os passos iniciais da Teoria dos Jogos (ver, por exemplo, Morgenstern [33]).

O surgimento da Programação Linear como ferramenta computacional para a solução de problemas complexos presentes em áreas como Indústria e Economia aconteceu definitivamente em 1947, quando G.B. Dantzig desenvolveu, dentro do Projeto SCOOP (Scientific Computation of Optimum Programs), o método computacional simplexo para a obtenção da solução de um problema linear. Neste mesmo ano, J. von Neumann apresentou o primeiro estudo sobre a Teoria da Dualidade [41], outra ferramenta importante para a construção de algoritmos que visam à solução de problemas lineares.

Em termos de teoria voltada a aplicações em Economia e Indústria, a Programação Linear avançou significativamente a partir do modelo econômico geral proposto por T.C. Koopmans em 1948 (ver, por exemplo, Koopmans [31]). Kantorovich também contribuiu com o desenvolvimento da teoria econômica por meio de um artigo escrito juntamente com M.K. Gavurin em 1949 [26] no qual apresentou pela primeira vez a teoria do problema de transbordo, que levaria anos mais tarde à formulação do problema de Kantorovich-Rubinstein (vide Seção 2.2). Outro trabalho relevante de Kantorovich foi um livro voltado para economistas e que foi publicado em 1959 [24]. Kantorovich e Koopmans receberam conjuntamente o Prêmio Nobel de Ciências Econômicas em 1975 por suas contribuições.

Curiosamente, os primeiros trabalhos de Kantorovich em termos de Programação Linear (sua monografia de 1939 e o artigo com Gavurin de 1949) passaram a ser reconhecidos apenas a partir do final da década de 1950 devido à ausência, em ambos os trabalhos, de um método computacional completo que tornasse possível a aplicação da teoria exposta pelo autor. Sobre o assunto, Dantzig comentou em 1963 [5, p. 23]: "Se os trabalhos iniciais de Kantorovich tivessem sido apreciados à época em que foram apresentados pela primeira vez, provavelmente a Programação Linear estaria mais avançada nos tempos atuais. Contudo, seu trabalho inicial nesta área continuou desconhecido tanto na União Soviética como em qualquer outro lugar pelas últimas duas décadas enquanto que a Programação Linear tornou-se uma arte extremamente desenvolvida". 


\subsection{O problema do transporte}

Nesta seção, formularemos o problema do transporte ótimo em termos de Programação Linear. Este problema ganhou importância (principalmente em áreas como Economia e Logística) a partir do início da década de 1940, quando sua forma padrão foi introduzida em um artigo escrito em 1941 por Hitchcock [17]. Sua formulação tem origem no problema de transportar determinadas quantidades de um bem de consumo com o menor custo possível, como veremos a seguir.

Suponhamos que existam $M$ indústrias que produzem, respectivamente, $a_{1}, a_{2}, \ldots, a_{M}$ unidades de um determinado produto. Tais unidades devem ser transportadas para $N$ mercados consumidores que têm uma demanda de $b_{1}, b_{2}, \ldots, b_{N}$ unidades, respectivamente. Assumimos que a produção total é igual à demanda total, ou seja,

$$
\sum_{i=1}^{M} a_{i}=\sum_{j=1}^{N} b_{j}
$$

Por hipótese, todos os números $a_{i}$ e $b_{j}$ são não-negativos e conhecidos. Também são dados valores $c_{i j} \geq 0, i=1, \ldots, M$ e $j=1, \ldots, N$, onde $c_{i j}$ representa o custo por unidade transportada da indústria $i$ para o mercado consumidor $j$. O problema do transporte consiste em obter valores $q_{i j} \geq 0$, $i=1, \ldots, M$ e $j=1, \ldots, N$ tais que o custo total de transporte

$$
\sum_{i=1}^{M} \sum_{j=1}^{N} c_{i j} q_{i j}
$$

seja mínimo. Evidentemente, $q_{i j}$ é a quantidade de unidades do produto em questão que deve ser transportada da indústria $i$ para o mercado $j$. Além disso, os valores $q_{i j}$ devem satisfazer as seguintes restrições lineares:

$$
\sum_{j=1}^{N} q_{i j}=a_{i} \quad \text { para todo } i=1, \ldots, M
$$

e

$$
\sum_{i=1}^{M} q_{i j}=b_{j} \quad \text { para todo } j=1, \ldots, N .
$$

Observe que, nessas condições, o problema do transporte enunciado acima equivale ao problema 
de Monge-Kantorovich no caso finito quando definimos as medidas de probabilidade

$$
\mu(i)=\frac{a_{i}}{\sum_{i=1}^{M} a_{i}}, i=1, \ldots, M
$$

$\mathrm{e}$

$$
\nu(j)=\frac{b_{j}}{\sum_{j=1}^{N} b_{j}}, j=1, \ldots, N .
$$

De acordo com a Teoria da Dualidade, o problema do transporte (problema primal) tem seu respectivo problema dual, que consiste em obter vetores $\mathbf{u}=\left(u_{1}, \ldots, u_{M}\right)$ e $\mathbf{v}=\left(v_{1}, \ldots, v_{N}\right)$, com $u_{i}, v_{j} \in \mathbb{R}$, tais que

$$
u_{i}+v_{j} \leq c_{i j} \text { para todo } i, j
$$

e que maximizem a função objetivo

$$
D(\mathbf{u}, \mathbf{v})=\sum_{i=1}^{M} u_{i} a_{i}+\sum_{j=1}^{N} v_{j} b_{j}
$$

Uma observação importante a ser feita é que ambos os problemas são possíveis de ser solucionados, pois o conjunto de valores $q_{i j}$ que satisfazem (5.3) e (5.4) não é vazio (basta tomar, por exemplo, $\left.q_{i j}=\frac{a_{i} b_{j}}{\sum_{i=1}^{M} a_{i}}\right)$, assim como o conjunto de valores $u_{i}$ e $v_{j}$ que satisfazem $(5.5)\left(u_{i}=v_{j}=0\right)$. O próximo Teorema (Dantzig [5, p. 129]) determina a equivalência entre os dois problemas:

Teorema 5.2.1 (Teorema da Dualidade para o problema do transporte.) Ambos os problemas primal e dual relativos ao problema do transporte possuem uma solução ótima. Além disso, os dois problemas são equivalentes, ou seja,

$$
\min \left\{\sum_{i=1}^{M} \sum_{j=1}^{N} c_{i j} q_{i j}\right\}=\max _{\mathbf{u}, \mathbf{v}} D(\mathbf{u}, \mathbf{v})
$$

Este teorema assume um papel importante na construção de algoritmos que determinam a solução ótima para o problema do transporte e que são uma alternativa ao algoritmo simplexo que, baseado na teoria do método simplexo, é uma das ferramentas mais utilizadas para computar soluções ótimas de problemas lineares que possuem um número elevado de restrições lineares.

É interessante observar que em 1939, dois anos antes da publicação do artigo de Hitchcock, Kantorovich mostrou que "uma classe de problemas (que posteriormente mostrou-se relaciondada 
com a forma padrão do problema do transporte) apresenta uma variedade de aplicações ligadas à alocação de tarefas para máquinas cujos custos e taxas de produção variam por tarefa e tipo de máquina" (Dantzig [5, p. 299]).

\subsection{Uma sugestão de algoritmo}

O problema do transporte pode ser solucionado por meio do método simplexo. Entretanto, o algoritmo simplexo para este problema requer uma matriz de restrições de $M+N$ linhas e $M N$ colunas. Uma alternativa é utilizar um algoritmo mais eficiente baseado na idéia proposta por Dantzig [5, Capítulo 14]. Tal algoritmo pode ser descrito em termos da seguinte tabela de transporte ${ }^{2}$ com $M$ linhas e $N$ colunas:

\begin{tabular}{|c|c|c|c|}
\hline$c_{11}$ & $\begin{array}{lll}c_{12} & & \\
& & \\
& q_{12} \\
& \end{array}$ & . & $\begin{array}{ll}c_{1 N} & \\
& q_{1 N}\end{array}$ \\
\hline$c_{21}$ & $\begin{array}{lll}c_{22} & & \\
& \\
& q_{22} \\
& \end{array}$ & $\ldots$ & $\begin{array}{ll}c_{2 N} & \\
& q_{1 N}\end{array}$ \\
\hline$\vdots$ & $\vdots$ & $\ddots$ & : \\
\hline$c_{M 1}$ & $\begin{array}{ll}c_{M 2} & \\
& \\
& q_{M 2}\end{array}$ & $\cdots$ & $\begin{array}{l}c_{M N} \\
\\
\\
\\
q_{M N}\end{array}$ \\
\hline
\end{tabular}

A casela $(i, j)$, localizada na $i$-ésima linha e $j$-ésima coluna da tabela, contém o respectivo custo $c_{i j}$ no canto superior esquerdo e, no canto inferior direito, o valor $q_{i j}$ a ser determinado. As somas marginais, $a_{i}$ e $b_{j}$, estão fixadas nos cantos direito e inferior da tabela, respectivamente. Antes de descrevermos o algoritmo, apresentamos a seguinte

Definição 5.3.1 Dizemos que $\left\{\bar{q}_{i j} \geq 0, i=1, \ldots, M\right.$ e $\left.j=1, \ldots, N\right\}$ é uma solução básica do problema de transporte se satisfaz as restrições (5.3) e (5.4).

O algoritmo consiste em três partes:

P1. Determinar uma solução básica $\left\{q_{i j}\right\}$.

\footnotetext{
${ }^{2}$ Traduzido do inglês, transportation array.
} 
P2. Testar se essa solução é ótima.

P3. Se o teste funcionar, então $\left\{q_{i j}\right\}$ é solução ótima. Caso contrário, obter uma solução básica melhor que a anterior e repetir P2.

Na primeira etapa do algoritmo, obtemos uma solução básica inicial a partir de alguma regra que leve em conta o valor máximo que cada $q_{i j}$ pode assumir. Dentre as regras mais utilizadas, destaca-se a regra do custo mínimo ${ }^{3}$, que consiste basicamente em tentar atribuir valor máximo para os $q_{i j}$ que detêm os menores custos. A seguir, aplicamos a segunda parte do algoritmo obtendo $u_{i}$ e $v_{j}$ que satisfaçam (5.5): se tais valores existirem, então a solução básica é uma solução ótima; caso contrário, "melhoramos" a solução básica anterior de modo que a nova solução básica tenha custo total menor ou igual que o da solução anterior e testamos se esta solução é ótima de forma análoga. Discutiremos cada etapa do algoritmo no caso geral do problema do transporte e, posteriormente, apresentaremos um exemplo de aplicação do algoritmo no caso específico - a versão finita do problema de Monge-Kantorovich.

\subsubsection{A regra do custo mínimo}

Uma forma de obter uma solução ótima inicial é escolher primeiramente as caselas com os menores custos. Esta regra é conhecida como regra do custo minimo, que consiste nos seguintes passos:

(a) Escolha a casela com o menor custo $((k, l)$, por exemplo). Se houver mais de uma casela, escolha uma delas aleatoriamente.

(b) Atribua a $q_{k l}$ o maior valor possível, ou seja, $q_{k l}=\min \left\{a_{k}, b_{l}\right\}$. Cada elemento $q_{k l}$ definido desta maneira é chamado de variável básica.

(c) Podemos ter três situações diferentes:

- Se $a_{k}<b_{l}$, faça $q_{k j}=0$ para todo $j \neq l$. Observe que, nestas condições, a restrição da $k$-ésima linha está satisfeita $\left(\sum_{j=1}^{N} q_{k j}=a_{k}\right)$. A seguir, delete a $k$-ésima linha e reduza o valor de $b_{l}$ para $\left(b_{l}-a_{k}\right)$. Desta forma, a tabela de transporte fica reduzida a uma tabela com $M-1$ linhas e $N$ colunas.

- Se $a_{k}>b_{l}$, faça $q_{i l}=0$ para todo $i \neq k$. Analogamente, observe que a restrição da $l$-ésima coluna está satisfeita $\left(\sum_{i=1}^{M} q_{i l}=b_{l}\right)$. A seguir, delete a l-ésima coluna e reduza o valor

\footnotetext{
${ }^{3}$ Do inglês, least-cost rule.
} 
de $a_{k}$ para $\left(a_{k}-b_{l}\right)$. Desta forma, a tabela de transporte fica reduzida a uma tabela com $M$ linhas e $N-1$ colunas.

- Se $a_{k}=b_{l}$, delete a $k$-ésima linha ou a l-ésima coluna, mas não ambas. Entretanto, se a tabela tiver algumas colunas mas apenas uma linha, então delete a $k$-ésima linha; da mesma forma, se a tabela tiver algumas linhas mas apenas uma coluna, delete a $l$-ésima coluna.

(d) A partir da tabela reduzida, escolha a casela com o menor custo, seguindo o critério do passo (a), e repita os passos (b) e (c) até que a última casela disponível esteja preenchida com uma variável básica e então delete a respectiva linha e a respectiva coluna.

Observe que:

- O número total de variáveis básicas é $M+N-1$, uma vez que deletamos uma linha ou coluna para cada variável básica que é determinada, exceto na última variável básica, para a qual ambas são deletadas. Como há $M+N$ linhas ou colunas, há exatamente $M+N-1$ variáveis básicas.

- Nenhuma tabela reduzida com $r$ linhas e c colunas contém mais do que $r+c-1$ variáveis básicas, pois no máximo uma variável básica é deletada dessa tabela por cada uma de suas linhas e colunas, e a tabela é totalmente deletada quando $r+c-1$ de suas linhas e colunas são deletadas.

- Como conseqüência das duas observações anteriores, qualquer conjunto de variáveis básicas pode ser obtido pela regra do custo mínimo.

\subsubsection{Testando se uma solução é ótima}

Dada uma solução básica $\left\{q_{i j}, i=1, \ldots, M\right.$ e $\left.j=1, \ldots, N\right\}$, testaremos se essa solução é ótima. A proposta é resolver as equações da forma

$$
u_{i}+v_{j}=c_{i j}
$$

para todas as caselas $(i, j)$ que contêm variáveis básicas. O sistema linear possui $M+N-1$ equações (referente ao total de variáveis básicas) e $M+N$ incógnitas. Portanto, uma das incógnitas pode ser 
fixada (por exemplo, igual a zero) e usamos (5.8) para obter as outras. Uma vez que determinamos as variáveis duais $u_{i}$ e $v_{j}$, devemos verificar se

$$
u_{i^{\prime}}+v_{j^{\prime}} \leq c_{i^{\prime} j^{\prime}}
$$

para todas as caselas $\left(i^{\prime}, j^{\prime}\right)$ que não têm variáveis básicas. Se (5.9) valer para todo $\left(i^{\prime}, j^{\prime}\right)$, então $\left\{q_{i j}\right\}$ é uma solução ótima para o problema de transporte (problema primal):

$$
\min \left\{\sum_{i=1}^{M} \sum_{j=1}^{N} c_{i j} q_{i j}\right\}
$$

e as seqüências $\left\{u_{i}\right\}$ e $\left\{v_{j}\right\}$ determinam a solução do problema dual:

$$
\max \left\{\sum_{i=1}^{M} u_{i} a_{i}+\sum_{j=1}^{N} v_{j} b_{j}\right\}
$$

Além disso, pelo Teorema da Dualidade, garante-se que

$$
\min \left\{\sum_{i=1}^{M} \sum_{j=1}^{N} c_{i j} q_{i j}\right\}=\max \left\{\sum_{i=1}^{M} u_{i} a_{i}+\sum_{j=1}^{N} v_{j} b_{j}\right\} .
$$

Se existir pelo menos uma casela $\left(i_{0}, j_{0}\right)$ tal que $u_{i_{0}}+v_{j_{0}}>c_{i_{0} j_{0}}$, então $\left\{q_{i j}\right\}$ não é uma solução ótima para o problema de transporte. Neste caso, devemos fazer uma etapa de melhoria na solução básica de modo que o custo total seja reduzido.

\subsubsection{Melhorando uma solução básica}

Suponha que testamos a optimalidade de uma solução básica $\left\{q_{i j}\right\}$ por meio das variáveis duais $u_{i}$ e $v_{j}$ e verificamos que tal solução não é ótima. Logo, devemos construir uma nova solução básica $\left\{q_{i j}^{\prime}\right\}$ que tenha custo total menor ou igual que o custo total da solução básica original. Para isso, tomemos os seguintes passos:

(a') Escolha aleatoriamente uma casela $\left(i_{0}, j_{0}\right)$ tal que $u_{i_{0}}+v_{j_{0}}>c_{i_{0} j_{0}}$ e defina $q_{i_{0} j_{0}}=\theta$, mas mantenha as restrições de linha e de coluna somando e subtraindo $\theta$ das variáveis básicas apropriadas. 
(b') Escolha $\theta$ como sendo o menor valor das variáveis nas caselas em que subtraiu-se $\theta$.

(c') Transforme $q_{i_{0} j_{0}}$ em uma variável básica e escolha uma das variáveis básicas que assumiram o valor zero após a subtração de $\theta$ e transforme-a em não-básica.

Seja $\left\{q_{i j}^{\prime}\right\}$ a nova solução básica obtida a partir desses três passos. Observe que podemos testar se essa nova solução básica é uma solução ótima, uma vez que a mesma possui $M+N-1$ variáveis básicas.

Vamos comparar os custos totais das duas soluções básicas:

$$
\Delta=\sum_{i=1}^{M} \sum_{j=1}^{N} c_{i j} q_{i j}-\sum_{i=1}^{M} \sum_{j=1}^{N} c_{i j} q_{i j}^{\prime}=\theta \sum^{+} c_{i j}-\theta \sum^{-} c_{i j}
$$

onde $\sum^{+}$é a soma sobre as caselas para as quais $\theta$ foi adicionado e $\sum^{-}$é a soma sobre todas as caselas para as quais $\theta$ foi subtraído. Uma vez que $c_{i j}=u_{i}+v_{j}$ para todos estas caselas exceto para a nova casela $\left(i_{0}, j_{0}\right)$, podemos escrever

$$
\Delta=\theta\left(u_{i_{0}}+v_{j_{0}}-c_{i_{0} j_{0}}\right)
$$

porque cada $u_{i}$ e $v_{j}$ que é somado também é subtraído. Observe que $\Delta$ nunca é negativo e, mais do que isso, $\Delta>0$ se $\theta>0$.

É possível que $\theta=0$ e, neste caso, a nova solução básica não diminui estritamente o custo total, mas esta nova solução pode ser melhorada de forma análoga e, posteriormente, pode-se realizar um novo teste para verificar se é uma solução ótima.

\subsubsection{Exemplo de aplicação do algoritmo}

Finalmente, daremos um exemplo de aplicação do algoritmo que discutimos nesta seção. Consideramos o caso finito do problema de Monge-Kantorovich tomando $S=\{1,2,3,4\}(M=N=4)$, $c(x, y)=|x-y|$ para $x, y \in S$ e as distribuições marginais $\mu$ e $\nu$ são dadas por

$$
\begin{gathered}
\mu(1)=0.30, \mu(2)=0.15, \mu(3)=0.20 \text { e } \mu(4)=0.35 \\
\nu(1)=0.10, \nu(2)=0.05, \nu(3)=0.20 \text { e } \nu(4)=0.65
\end{gathered}
$$

Seja $Q$ um acoplamento de $\mu$ e $\nu$. Para a implementação do algoritmo, definimos para todo $i, j \in S$ 
as quantidades $a_{i}=\mu(i), b_{j}=\nu(j), c_{i j}=|i-j|$ e $q_{i j}=Q(i, j)$. Com isso, temos a seguinte tabela de transporte inicial:

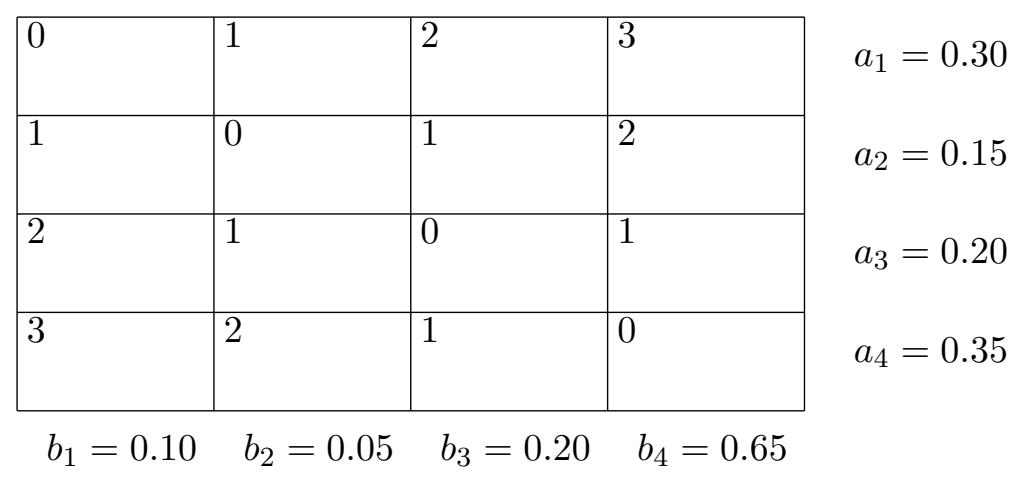

A partir da tabela acima, iniciamos o algoritmo:

Encontrar uma solução básica. Utilizaremos a regra do custo mínimo para obtermos uma solução básica inicial. Em primeiro lugar, observamos que o menor custo é igual a 0. Como quatro caselas detêm o menor custo, escolhemos uma delas aleatoriamente para iniciar o algoritmo, de acordo com o passo (a). Escolhemos a casela $(1,1)$ e definimos

$$
q_{11}=\min \left\{a_{1}, b_{1}\right\}=\min \{0.30,0.10\}=0.10
$$

Como $a_{1}>b_{1}$, fazemos $q_{21}=q_{31}=q_{41}=0$ e, portanto, a restrição da primeira coluna está satisfeita. Além disso, marcamos $q_{11}=0.10$ em negrito, indicando que é variável básica, e atualizamos o valor de $a_{1}$ para

$$
a_{1}=0.30-0.10=0.20
$$

e o valor de $b_{1}$ para

$$
b_{1}=0
$$

indicando que não há mais massa de probabilidade a ser distribuída na coluna. Analogamente ao que foi feito com $q_{11}$, destacamos $a_{1}$ e $b_{1}$ em negrito para indicar que seu valores também foram modificados. A tabela de transporte resultante desta primeira iteração é apresentada a seguir. 


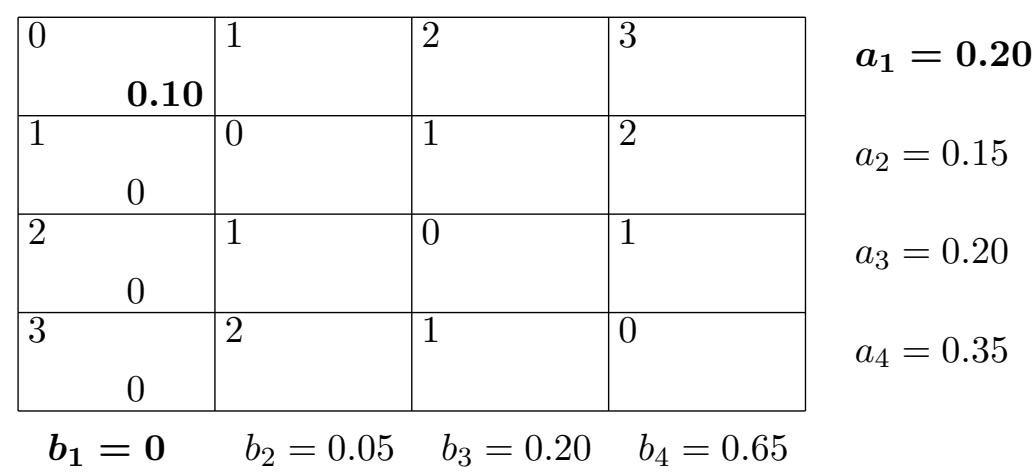

A seguir, repetimos o procedimento para as caselas $(2,2),(3,3)$ e $(4,4)$, respectivamente. Como $a_{3}=b_{3}$, escolhemos cancelar a terceira coluna por haver mais elementos a serem cancelados do que a terceira linha. A tabela fica da seguinte forma:

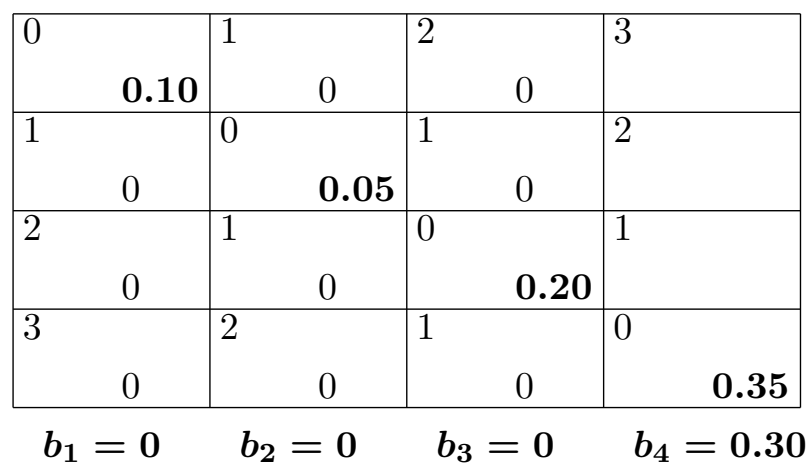

$a_{1}=0.20$

$a_{2}=0.10$

$a_{3}=0$

$a_{4}=0$

Todas as caselas com custo 0 foram preenchidas. Passamos agora para a casela com o segundo menor custo (que é igual a 1 ), que é $(3,4)$. Para esta casela, definimos

$$
q_{34}=\min \left\{a_{3}, b_{4}\right\}=\min \{0,0.30\}=0 .
$$

Destacamos $q_{34}$ como variável básica e atualizamos os valor de $b_{4}$ para $b_{4}=0.30$. Da mesma forma, obtemos a nova variável básica $q_{24}=0.10$ (a casela $(2,4)$ detém o terceiro menor custo, que é igual a 2 ) e atualizamos novamente o valores de $a_{2}$ e $b_{4}$ para $a_{2}=0$ e $b_{4}=0.20$. Finalmente, resta determinar a última variável básica $q_{14}=0.20$ e atualizar $a_{1}$ e $b_{4}$ por $a_{1}=b_{4}=0$. Como $(1,4)$ foi a última casela a ser preenchida, a primeira parte do algoritmo está encerrada. A tabela de transporte final, com a 
primeira solução básica, é apresentada a seguir. Observe que há exatamente $M+N-1=7$ variáveis básicas na solução básica obtida.

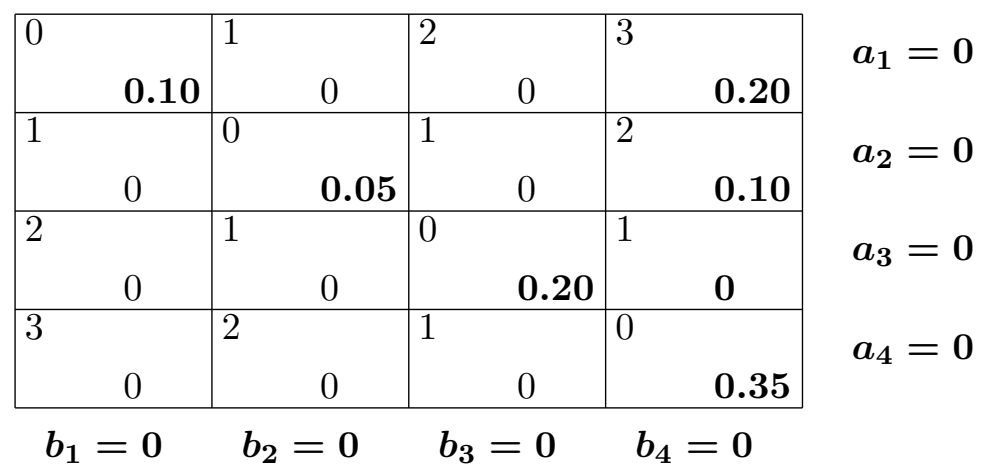

Testar se a solução é ótima. Dada a solução básica obtida na primeira parte do algoritmo, determinaremos as variáveis duais $u_{i}$ e $v_{j}$, com o objetivo de testar se a mesma também é solução ótima do problema.

Em primeiro lugar, observamos que a quarta coluna possui 4 caselas com variáveis básicas. Então, escolhemos arbitrariamente $v_{4}=0$ e, como conseqüência imediata de (5.8), segue que $u_{1}=3, u_{2}=2$, $u_{3}=1$ e $u_{4}=0$. Da casela $(1,1)$, temos que $v_{1}=-3$. De maneira análoga, a partir das caselas $(2,2)$ e $(3,3)$, respectivamente, temos que $v_{2}=-2$ e $v_{3}=-1$.

O próximo passo é verificar se (5.9) vale para todas as caselas $(i . j)$ sem variáveis básicas:

$$
\begin{array}{r}
u_{1}+v_{2}=1 \leq c_{12}=1 \\
u_{1}+v_{3}=2 \leq c_{13}=2 \\
u_{2}+v_{1}=-1 \leq c_{21}=1 \\
u_{2}+v_{3}=1 \leq c_{23}=1 \\
u_{3}+v_{1}=-2 \leq c_{31}=2 \\
u_{3}+v_{2}=-1 \leq c_{32}=1 \\
u_{4}+v_{1}=-3 \leq c_{41}=3 \\
u_{4}+v_{2}=-2 \leq c_{42}=2 \\
u_{4}+v_{3}=-1 \leq c_{43}=1
\end{array}
$$


Como todas as desigualdades estão satisfeitas, a solução básica obtida é uma solução ótima do problema de Monge-Kantorovich, cujo mínimo é dado por

$$
K(\mu, \nu)=\sum_{i=1}^{4} \sum_{j=1}^{4} c_{i j} q_{i j}=2 \times 0.10+3 \times 0.20=0.80
$$

A título de curiosidade, o máximo da função objetivo do problema dual é dado por

$$
D(\mathbf{u}, \mathbf{v})=\sum_{i=1}^{4} u_{i} a_{i}+\sum_{j=1}^{4} v_{j} b_{j}=1.40-0.60=0.80,
$$

ou seja, $K(\mu, \nu)=D(\mathbf{u}, \mathbf{v})$, o que está de acordo com o Teorema da Dualidade. 


\section{Capítulo 6}

\section{Considerações finais}

O problema do transporte ótimo de Monge-Kantorovich, desde a década de 1940, destaca-se como um dos principais objetos de estudo em várias áreas do conhecimento, com destaque para Teoria das Probabilidades, Estatística, Análise Funcional e Programação Linear. Nesta dissertação, apresentamos alguns aspectos relacionados, principalmente, com Teoria das Probabilidades - discutimos sobre a construção de acoplamentos que solucionam o problema no caso finito, e Programação Linear citando um algoritmo que chega a uma solução ótima do problema.

Com base no que foi apresentado, enunciamos duas sugestões para pesquisas futuras relacionadas com o problema de Monge-Kantorovich:

- Estudar o problema de Monge-Kantorovich no caso finito quando as distribuições marginais $\mu$ e $\nu$ correspondem a distribuições estacionárias de cadeias estocásticas de memória de alcance variável;

- Estabelecer resultados para a obtenção de soluções para o problema de Monge-Kantorovich em espaços produto finitos quando a função custo $c(x, y)$ é o produto de funções custo definidas nos espaços marginais, ou seja, $c(x, y)=\prod_{i=1}^{n} c_{i}\left(x_{i}, y_{i}\right)$. 


\section{Referências Bibliográficas}

[1] L. Ambrosio, Y. Brenier, G. Buttazzo, L.A. Caffarelli e C. Villani, Optimal tranportation and applications, Lecture Notes in Mathematics, vol. 1813, Springer-Verlag, Berlin, 2003, Lectures from the C.I.M.E. Summer School held in Martina Franca, September 2-8, 2001, Edited by Caffarelli and S. Salsa.

[2] E.J. Anderson e P. Nash, Linear Programming in Infinite Dimensional Spaces. Theory and Applications, Wiley, New York, 1987.

[3] P. Appell, Mémoire sur les déblai et les remblais des systeèmes continus ou discontinus, Mémoires présentés par divers Savants à l'Académie des Sciences de l'Institut de France 28 (1887), 1-208.

[4] E.R. Barnes e A.J. Hoffman, On transportation problems with upper bounds on leading rectangles, SIAM Journal of Algebraic and Discrete Methods 6 (1985), 487-496.

[5] G.B. Dantzig, Linear Programming and Extensions, Princeton University Press, Princeton, New Jersey, 1963.

[6] G.B. Dantzig e A.R. Ferguson, The allocation of aircraft to routes-an example of Linear Programming under uncertain demands, Management Science 3 (1956), 45-73.

[7] R.L. Dobrushin, Vlasov equations, Func. Anal. Appl. 13 (1979), 115-123.

[8] R.L. Dobrushin, Perturbation methods of the theory of Gibbsian fields, Lecture Notes in Math., vol. 1648, pp. 1-66, Springer, Berlin, 1996.

[9] D.C. Dowson e B.V. Landau, The Frechét distance between Multivariate Normal Distributions, Journal of Multivariate Analysis 12 (1982), 450-455.

[10] R.M. Dudley, Probabilities and metrics, Matematisk Institut, Aarhus Universitet, 1976, Convergence of laws on metric spaces, with a view to statistical testing, Lecture Notes Series, No. 45 .

[11] R.M Dudley, Real Analysis and Probability, Wadsworth \& Brooks-Cole, Pacific Grove, California, 1989. 
[12] R.M. Dudley e J. Neveu, On Kantorovich-Rubinstein theorems, 1981, (Transcrição).

[13] J. Fourier, Solution d'une question particulière du calcul des inégalités, 1826.

[14] M. Fréchet, Sur les tableaux de corrélation dont les marges sont donnés, Ann.Univ. de Lyon, Sciences 14 (1951), 53-77.

[15] C. Gini, La dissomiglianza, Matron 24 (1965), 309-331.

[16] N.E. Gretsky, J.M. Ostroy e W.R. Zame, The nonatomic assignment model, Journal of Economic Theory 2 (1992), 103-128.

[17] F.L. Hitchcock, The distribution of a product from several sources to numerous localities, J. Math. Phys. 20 (1941), 224-230.

[18] W. Hoeffding, The extrema of the expected value of a function of independent random variables, Annals of Mathematical Statistics 26 (1955), 268-275.

[19] A.J. Hoffman, On simple Linear Programming problems, Proceedings of Symposia in Pure Mathematics, vol. 7, pp. 317-327, Am. Math. Society, Providence, Rhode Island, 1961.

[20] M. Jirina e J. Nedoma, Minimax solution of a sampling inventory process, Applikace mathematiky 1 (1957), 296-314, In Czech.

[21] L.V. Kantorovich, Mathematical Methods in the Organization and Planning of Production, Publication House of The Leningrad State University (1939), Traduzido para o Inglês em Management Science 6, 1960, 366-422.

[22] L.V. Kantorovich, On the translocation of masses, Dokl. Akad. Nauk 37 (1942), no. 7-8, 227-229, Traduzido para o Inglês em J. of Math. Sciences 133 (2006), no. 4, 1381-1382.

[23] L.V. Kantorovich, On a problem of Monge, Uspekhi Mat. Nauk 3 (1948), no. 2, 225-226, Traduzido para o Inglês em J. of Math. Sciences 133 (2006), no. 4, p. 1383.

[24] L.V. Kantorovich, The Best Use of Economic Resources, Acad. of Sci., USSR, 1959. Traduzido para o Inglês em 1965.

[25] L.V. Kantorovich e G.P. Akilov, Functional Analysis in Normed Spaces, The MacMillan Company, New York, 1964.

[26] L.V. Kantorovich e M.K. Gavurin, The Application of Mathematical Methods to Problems of Freight Flow Analysis, Akad. Nauk (1949), 110-138.

[27] L.V. Kantorovich e G.S. Rubinstein, On a function space in certain extremal problems, Dokl. Akad. Nauk 115 (1957), no. 6, 1058-1061. 
[28] L.V. Kantorovich e G.S. Rubinstein, On the space of completely additive functions, Ser. Mat. Mekh. i Astron. 13 (1958), no. 7, 52-59.

[29] J.H.B. Kemperman, On the role of duality in the theory of moments, Semi-Infinite Programming and Applications 1981, vol. 215, pp. 63-92, Springer, 1983.

[30] J.H.B. Kemperman, Geometry of the moment problem, Proceedings of Symposia im Applied Mathematics, vol. 27, pp. 16-53, American Mathematical Society, 1987.

[31] T.C. Koopmans, Activity Analysis of Production and Allocation, John Wiley \& Sons, Inc., New York, 1951.

[32] G. Monge, Mémoire sur la théorie des déblais et des remblais, 1781.

[33] O. Morgenstern, The Theory of Games, Sci. American 180 (1949), no. 5, 22-25.

[34] S.T. Rachev, Monge-Kantorovich mass transference problem and its stochastic apllications, Th. Prob. Appl. 24 (1984), no. 4, 647-676.

[35] S.T. Rachev, The problem of stability in queueing theory, Queueing Systems Theory and Applications 4 (1989), 287-318.

[36] S.T. Rachev, Probability Metrics and the Stability of Stochastic Models, Wiley, Chichester-New York, 1991.

[37] S.T. Rachev e L. Rüschendorf, Mass Transportation Problems, Volume I: Theory, Probability and its Applications, Springer, New York, 1998.

[38] L. Rüschendorf, Fréchet-bounds and their applications, Math. Appl., vol. 67, pp. 151-187, Kluwer Acad. Publ., Dordrecht, 1991.

[39] V. Strassen, The existence of probability measures with given marginals, Ann. of Math. Statistics 36 (1965), no. 2, 423-439.

[40] V.N. Sudakov, Geometric problems in the theory of infinite dimensional probability distributions, Proc. Steklov Inst. Math. 141 (1979), no. 2, 1-178.

[41] J. von Neumann, On a maximization problem, Institute for Advanced Study, Princeton, New Jersey, 1947, (Transcrição).

[42] L.N. Wasserstein, Markov processes over denumerable products of spaces describing large systems of automata, Problems of Information Transmission 5 (1969), no. 3, 47-52. 